\title{
Silk-Based Anisotropical 3D Biotextiles for Bone Regeneration
}

V.P. Ribeiro ${ }^{1,2}$, J. Silva-Correia ${ }^{1,2}$, A.I. Nascimento ${ }^{1,2}$, A. da Silva Morais ${ }^{1,2}$, A.P. Marques ${ }^{1,2}$, A.S.

Ribeiro $^{3}$, C.J. Silva ${ }^{3}$, G. Bonifácio ${ }^{4}$, R.A. Sousa ${ }^{5}$, J.M. Oliveira ${ }^{1,2}$, A.L. Oliveira ${ }^{1,2,6 *}$, R.L. Reis ${ }^{1,2}$

${ }^{1}$ 3B's Research Group - Biomaterials, Biodegradables and Biomimetics, Universidade do Minho, Headquarters of the European Institute of Excellence on Tissue Engineering and Regenerative Medicine, AvePark - Parque de Ciência e Tecnologia, Zona Industrial da Gandra, 4805-017 Barco, Portugal

${ }^{2}$ ICVS/3B's - PT Government Associated Laboratory, Braga/Guimarães, Portugal

${ }^{3}$ CeNTI - Centre for Nanotechnology and Smart Materials, Rua Fernando Mesquita, 4760-034 Vila Nova de Famalicão, Portugal

${ }^{4}$ CITEVE - Technological Center for Textile and Clothing Industry, Rua Fernando Mesquita, 4760-034 Vila Nova de Famalicão, Portugal

${ }^{5}$ Stemmatters, Biotecnologia e Medicina Regenerativa SA, AvePark - Parque de Ciência e Tecnologia, Zona Industrial da Gandra, 4805-017 Barco, Portugal

${ }^{6}$ CBQF-Center for Biotechnology and Fine Chemistry, Portuguese Catholic University, Rua Arquiteto Lobão Vital, 4202-401 Porto, Portugal

\section{"Corresponding author:}

Ana Leite Oliveira School of Biotechnology

Portuguese Catholic University

CBQF - Center for Biotechnology and Fine Chemistry

Rua Arquiteto Lobão Vital Apartado 2511 4202-401 Porto

$\mathrm{Ph}+351225580118$ Ext. 1271

e-mail addresses: aloliveira@porto.ucp.pt; rgreis@dep.uminho.pt 


\section{Abstract}

Bone loss in the craniofacial complex can been treated using several conventional therapeutic strategies that face many obstacles and limitations. In this work, novel three-dimensional (3D) biotextile architectures were developed as a possible strategy for flat bone regeneration applications. As a fully automated processing route, this strategy as potential to be easily industrialized. Silk fibroin (SF) yarns were processed into weft-knitted fabrics spaced by a monofilament of polyethylene terephthalate (PET). A comparative study with a similar 3D structure made entirely of PET was established. Highly porous scaffolds with homogeneous pore distribution were observed using micro-computed tomography analysis. The wet state dynamic mechanical analysis revealed a storage modulus In the frequency range tested, the storage modulus values obtained for SF-PET scaffolds were higher than for the PET scaffolds. Human adipose-derived stem cells (hASCs) cultured on the SF-PET spacer structures showed the typical pattern for ALP activity under osteogenic culture conditions. Osteogenic differentiation of hASCs on SF-PET and PET constructs was also observed by extracellular matrix mineralization and expression of osteogenic-related markers (osteocalcin, osteopontin and collagen type I) after 28 days of osteogenic culture, in comparison to the control basal medium. The quantification of convergent macroscopic blood vessels toward the scaffolds by a chick chorioallantoic membrane assay, showed higher angiogenic response induced by the SF-PET textile scaffolds than PET structures and gelatin sponge controls. Subcutaneous implantation in CD-1 mice revealed tissue ingrowth's accompanied by blood vessels infiltration in both spacer constructs. The structural adaptability of textile structures combined to the structural similarities of the 3D knitted spacer fabrics to craniofacial bone tissue and achieved biologial performance, make these scaffolds a possible solution for tissue engineering approaches in this area.

\section{Keywords}

biotextile; textile; silk fibroin; knitted spacer fabrics; human adipose-derived stem cells; craniofacial bone tissue engineering 


\section{Introduction}

Craniofacial critical-sized bone defects can be caused by several conditions, including tumor excisions, trauma, congenital malformations, degenerative skeletal disorders and advanced bone resorption due to teeth extraction [1]. More than 1.5 million bone grafts, including autologous or allogenic grafts, xenografts or alloplastic graft materials, are performed every year in the USA and the maxillofacial region is the most affected with over then three-quarters of surgical interventions every year [2]. Nevertheless, these standard clinical practices still present significant limitations in the treatment of craniofacial defects, including the need for complex-shaped bones that may complicate the surgical process. The use of metal implants as bone substitutes came as a possible solution in some cases, however, the frequent foreign body reaction makes this solution impossible as a lifetime treatment [3]. In this sense, the craniofacial bone regeneration involving scaffolding strategies and tissue engineering (TE) approaches have emerged to fulfil the gaps presented by the current therapeutic modalities [4].

Flat bones, as those from the craniofacial region, possess an anisotropic architecture composed of two nearly parallel plates of compact bone enclosing a layer of trabecular bone [2]. Scaffolds designed for craniofacial TE must contain such specificities, two dense external layers to sustain the masticatory forces at the maxillofacial region or to protect the constant brain pressures, and a porous spongy-like inner part to facilitate scaffolds adaptability and integration from the surrounding tissues, improving neotissue formation and vascular invasion [5].

Fibre-based networks with high surface area and interconnectivity have been proven to be particularly interesting to prepare polymeric porous 3D scaffolds for TE applications [6, 7]. Computer assisted textile-based technologies constitute an attractive route for the production of more complex fibrous three-dimensional (3D) scaffolds, based on automated pre-established techniques with high reproducibility and controlled properties [8]. Different textile production systems, including stitching, braided, woven, non-woven and knitted have already been proposed as scaffolding strategies. For example, Moutos et al. [9] developed 3D woven composite scaffolds for cartilage TE applications by combining a chondrocyte-hydrogel mixture into tissue constructs, to achieve improved mechanical properties. Liu et al. [10] combined microporous silk sponges with knitted silk mesh fo specifc ligament TE, and Chen et al. [11] developed a new practical ligament scaffold based on the synergistic incorporation of a plain knitted silk structure and a collagen matrix. The application of these textile production systems have also been proposed for bone TE applications [12, 13]. Knitted textile technologies are particularly useful for the fabrication of 3D fibre-based structures, since they allow for producing structures with 
complex shapes using rapid manufacturing processes. Moreover, they can be successfully adjusted to the use of several types of fibre materials, present controlled internal porosity, enhanced flexibility and high resistance to deformation, which makes this technique particularly desired for TE applications [14-16]. The resulting structural similarities to the anisotropic architecture of flat bone, makes the 3D knitted spacer fabrics a possible solution for craniofacial bone scaffolding strategies.

Synthetic materials and ceramic implants composed of hydroxyapatite $(\mathrm{HA})$ and calcium phosphate are still the most used, especially due to the high chemical similarity to bone [17]. However, natural-based materials have gained much attention as craniofacial bone scaffolding systems, showing high osteoconductivity and controlled degradability $[18,19]$. Natural silk fibroin (SF)-based materials have been successfully used for bone TE applications [20]. For example, Meinel and co-workers [21] have demonstrated well the feasibility of silk-based implants with engineered bone for the regeneration of bone tissue as a mechanically stable option. More recently Mandal et al. [22] developed a microfiber-reinforced silk scaffolds with high compressive strength as matrices for load-bearing bone applications with adequate in vivo response. The repair and regeneration of cranial defects have also been addressed using silk fibroin nanofibrous membranes [23] and porous scaffolds $[24,25]$.

Human adipose-derived stem cells (hASCs), isolated from the stromal vascular fraction (SVF) of adipose tissue have emerged as a viable possibility for bone regeneration applications due to the comparable features to bone marrow stromal cells (BMSCs), including similar morphology, differentiation capacity and phenotype [26]. In addition, they hold the advantage of being easily harvested from the adipose tissue in relative abundance and with less morbidity at the donor site [27]. There have been many recent reports indicating that hASCs can differentiate along multiple lineage pathways, including into osteogenic lineages when appropriatly stimulated $[26,28,29]$. It is also important to consider that the biological environment involves different cell interactions, which justify the use of a source of cells that can be used for several differentiation pathways [30]. Thus, the use of hASCs is relevant not only for bone TE but for other TE applications and clinical approaches. In a previous study, Correia et al. [20] were able to successfully explore the potential of hASC as a single cell source for osteogenic and endothelial differentiation and the assembly of bone and vascular compartments within the same scaffold, using silk porous matrices.

In the present study, a highly anisotropic scaffold was designed for bone TE applications, using natural silk yarns processed for the first time to form 3D knitted spacer fabrics. This structure is composed of two dense knitted sheets of SF spaced by a monofilament of polyethylene terephthalate (PET), the so-called SF-PET 
scaffolds, as a viable model for flat bone regeneration. A comparative analysis was performed using a knitted spacer scaffold entirely composed of PET. The morphology and 3D structure of the developed scaffolds were evaluated by scanning electron microscopy (SEM) and micro-computed tomography (micro-CT). The dynamic mechanical analysis (DMA) and static mechanical properties were also analyzed. Osteogenic differentiation of human adipose derived stem cells (hASCs) seeded in the SF-PET and PET scaffolds was also evaluated up to 28 days of in vitro culture. The in vivo biological response and agiogenic potential were investigated in a chick choroallontoic membrane assay and by subcutaneous implantation in a mice model.

\section{Materials and methods}

\subsection{Materials}

Bombyx mori cocoons were supplied by the Portuguese Association of Parents and Friends of Mentally Disabled Citizens (APPACDM, Castelo Branco, Portugal).

\subsection{Production of the textile-based scaffolds}

Raw silk yarns were processed from the combination of 4 to 8 cocoons into threads of raw silk. The reeled silk was then combined by twisting into a silk yarn, used to produce the 3D knitted spacer fabric by weftknitting technology (Terrot, UP472T model, Germany). A 100D/1Fpolyester monofilament (from Putian Shuangyan Chemical Fiber Co., Ltd.) was used on the knitting machine, to form the SF-PET spacer fabric. The $100 \%$ PET warp-knitted spacer fabric was produced on a warp-knitting machine (COMEZ, model DNB/EL-800-8B), with a working width of $812.8 \mathrm{~mm}$ and stitch density between $1-40$ stitches $/ \mathrm{cm}$. The same monofilament was used to interconnect the outer PET knitts. The polyester based filaments used in this spacer fabric construction were produced in a pilot spinning line (from Hills Inc.) using Polyethylene terephthalate Type RT52, with 0.64 of intrinsic viscosity (supplied by INVISTA ${ }^{\text {TM }}$ Resins \& Fibers) as raw material. The temperatures of the spinning line were maintained between $250^{\circ} \mathrm{C}$ and $270^{\circ} \mathrm{C}$, the spinning speed was $1500 \mathrm{~m} / \mathrm{min}$ for a throughput of $1.3 \mathrm{Kg} / \mathrm{h}$ with a draw ratio of 3.75 . A yarn with 36 continuous filaments was obtained, with 3.6 of denier per filament equivalent to about $15 \mu \mathrm{m}$ per filament.

Textile scaffolds (both SF-PET and PET) were first washed in a $0.15 \%(w / v)$ natural soap aqueous solution for 2 hours and then rinsed in distilled water to remove the impurities resulting from the manufacturing process. In this sense, SF-PET spacer fabrics underwent a subsequent purification process. Structures were boiled for 1 hour in a $0.03 \mathrm{M} \mathrm{Na}_{2} \mathrm{CO}_{3}$ solution (Sigma-Aldrich, Portugal), and then rinsed in boiling distilled water to ensure the full extraction of sericin. 


\subsection{Morphological characterization}

\subsubsection{Scanning electron microscopy}

Scanning Electron Microscopy (SEM) analysis was performed using a Leica Cambridge S360 (UK) to investigate the morphology of the produced spacer structures. All samples were sputter-coated with gold (Fisons Instruments, Sputter Coater SC502, UK) prior analysis and the micrographs were taken at an accelerating voltage of $15 \mathrm{kV}$ at different magnifications.

\subsubsection{Micro-computed tomography}

The microstructure of the developed spacer textile structures was evaluated using a high-resolution X-ray microtomography system Skyscan 1072 scanner (Skyscan, Belgium). The different materials were scanned both for the external sheets (Top) as for the internal monofilament (Bulk). SF-PET samples were scanned using a pixel size of $11.31 \mu \mathrm{m} \mathrm{x} / \mathrm{y} / \mathrm{z}$ and an X-ray source fixed at $41 \mathrm{keV}$ of energy and $215 \mu \mathrm{A}$ of current. For PET fabrics the pixel size used was $14.71 \mu \mathrm{m} x / y / z$ and the $X$-ray source was set at $40 \mathrm{keV}$ and $154 \mu \mathrm{A}$. Representative serial images in each data set (Top and Bulk) were transferred into binary images by using a dynamic threshold of 60-225 for SF-PET and 59-255 for PET fabrics. Then, the binary images were used for morphometric analysis (CT Analyzer v1.5.1.5, SkyScan, Belgium), which included the quantification of porosity, mean pore size and mean pore thickness. The 3D virtual models of the spacer structures were also created, visualized and registered using the image processing software CT-Vox and Data Viewer (both from SkyScan, Belgium). A minimum number of three specimens were used for the qualitative and quantitative microstructure evaluation.

\subsection{Mechanical properties}

\subsubsection{Compressive tests}

The mechanical behavior of the spacer textile structures was tested under quasi-static compression (dry state) using a Universal Testing Machine (Instron 4505, USA). SF-PET and PET specimens were cut into cuboid shapes of approximately $10 \times 10 \times 3 \mathrm{~mm}$ and $10 \times 10 \times 2.5 \mathrm{~mm}$, respectively. Textile structures were submitted to a pre-loaded of $1 \mathrm{kN}$ at room temperature (RT). The cross-head speed was fixed at $2 \mathrm{~mm} /$ minute and tests were run until a $60 \%$ deformation in specimen height was achieved. The elastic modulus $(E)$ was defined by the slope of the initial linear section of the stress-strain curve.

\subsubsection{Dynamic mechanical analysis}


The dynamic mechanical behavior of spacer textile structures was determined using a TRI-TEC8000B dynamic mechanical analyzer (Triton Technology, UK) in the compressive mode and measurements were carried out in hydrated state at $37^{\circ} \mathrm{C}$. The dimensions of the tested SF-PET and PET specimens were the same as for the compressive tests in dry state. Samples were always analyzed immersed in a liquid bath placed in a Teflon $\AA$ reservoir. Prior analysis, the specimens were immersed in a phosphate-buffered saline solution (PBS, pH 7.4) for 3 days, until equilibrium was reached $\left(37^{\circ} \mathrm{C}\right)$. Afterwards, the specimens were clamped in the DMA apparatus and kept immersed in the PBS solution during the measurements. After equilibration at $37{ }^{\circ} \mathrm{C}$, the DMA spectra were obtained in a frequency scan between 0.1 and $10 \mathrm{~Hz}$. The experiments were performed under the constant strain amplitude of $30 \mu \mathrm{m}$. Both storage modulus $(E)$ and loss factor (tan $\delta)$ were obtained in the frequency range. Before the analysis, a small pre-load was applied to each sample to ensure the complete contact of the structure surface with the compression plates, being the distance between plates equal for all tested samples. Three specimens of each group were tested.

\subsection{In vitro cell studies}

\subsection{1. hASCs isolation and expansion}

Human adipose-derived stem cells (hASCs) were isolated from subcutaneous adipose tissue samples obtained from liposuction procedures performed in patient's informed consent and using a protocol previously established with the Department of Plastic Surgery of Hospital da Prelada (Porto, Portugal). All the samples were processed within 24 hours after the liposuction procedure and human ASCs were enzymatically isolated from the subcutaneous adipose tissue, as previously described [27]. Human ASCs were expanded in basal medium consisting of MEM alpha medium ( $\alpha$-MEM; Life Technologies, Scotland) supplemented with sodium bicarbonate (Sigma-Aldrich, Australia), 10\% (v/v) fetal bovine serum (FBS; Biochrom AG, Germany) and 1\% (v/v) antibiotic/antimycotic solution (final concentration of penicillin 100 units $/ \mathrm{mL}$ and streptomycin $100 \mathrm{mg} / \mathrm{mL}$; Gibco, GB). Cells were cultured until confluence at $37{ }^{\circ} \mathrm{C}$ in an atmosphere of $5 \% \mathrm{CO}_{2}$ incubator, changing the culture medium every 2 days.

\subsubsection{Seeding and osteogenic differentiation of hASCs in the textile-based scaffolds}

Before the in vitro studies, SF-PET and PET textile scaffolds were cut into cylindrical shapes of approximately $10 \mathrm{~mm}$ diameter and $3 \mathrm{~mm}$ or $2.5 \mathrm{~mm}$ thickness, respectively, and sterilized by ethylene oxide. Before cell seeding, all samples were hydrated in PBS overnight in the $\mathrm{CO}_{2}$ incubator. In the following day, the hydrated textiles were transferred to 48-well suspension cell culture plates (Cellstar, Greiner Bio-One, Austria). Confluent hASCs (passage 4) were detached with TrypLE Express (1X) (Life Technologies, Carlsbad, CA, USA) and 
seeded in a $200 \mu \mathrm{L}$ cell suspension onto the SF-PET and PET spacer fabrics, at a density of $4 \times 10^{5}$ cells/scaffold and $3 \times 10^{5}$ cells/scaffold, respectively, according to scaffolds diameter and thickness. Each scaffold was kept in the $\mathrm{CO}_{2}$ incubator for 3 hours, and then $500 \mu \mathrm{L}$ of $\alpha-\mathrm{MEM}$ culture medium were added to each well. After 24 hours, cell culture medium was refreshed or changed to osteogenic differentiation medium (basal medium supplemented with $10^{-8} \mathrm{M}$ dexamethasone, $50 \mu \mathrm{g} / \mathrm{mL}$ ascorbic acid and $10 \mathrm{mM} \beta$ glycerophosphate). Samples were retrieved at different culture times $(1,7,14,21$ and 28 days) and the culture medium was changed every 2-3 days.

\subsubsection{Scanning electron microscopy and energy dispersive spectroscopy analysis}

Cell morphology and distribution were observed in the 3D textile scaffolds by SEM. Constructs were rinsed in PBS and fixed with a $2.5 \%(v / v)$ glutaraldehyde solution (Sigma-Aldrich, USA) for 1 hour at $4{ }^{\circ} \mathrm{C}$. Samples were further dehydrated through increasing series of ethanol concentrations $(30,50,70,90,100 \% \mathrm{v} / \mathrm{v})$ for 15 minutes, twice each concentration and left in hexamethylidisilazane (HMDS; Electron Microscopy Sciences, USA) overnight. Prior to SEM observation, all scaffolds were sputter-coated with gold and a graphite coating was used for energy dispersive spectroscopy (EDS; link-eXL-II) analysis to detect the elemental composition of the samples.

\subsubsection{DNA quantification}

The amount of double strained DNA (dsDNA), that is directly proportional with the cell number, was determined using a fluorimetric dsDNA quantification kit (PicoGreen, Molecular Probes, Invitrogen Corporation, USA), according to the manufacturer's instructions. After each culture time, samples were washed twice with PBS solution and kept in $1 \mathrm{~mL}$ of ultrapure water at $-80{ }^{\circ} \mathrm{C}$ until further analysis. Constructs were thawed at RT and sonicated for 1 hour to induce complete membrane lysis. Supernatant fluorescence was measured at an excitation wavelength of $485 / 20 \mathrm{~nm}$ and at an emission wavelength of 528/20 $\mathrm{nm}$, in a microplate reader (Synergy HT, BioTek Instruments, USA). The quantification of DNA was calculated according to a standard curve prepared with concentrations ranging between 0 and $2 \mu \mathrm{g} / \mathrm{mL}$, relating quantity of DNA and fluorescence intensity. Samples without cells were used as control.

\subsubsection{Alkaline phosphatase activity quantification}

The alkaline phosphatase (ALP) activity was determined on the same samples used for dsDNA quantification as a marker of ostegenic differentiation. ALP activity from constructs was quantified by the specific conversion of $p$ nitrophenyl phosphate (pNPP; Sigma-Aldrich, MO, USA) into p-nitrophenol (pNP). A buffer solution containing 
$0.2 \%(\mathrm{w} / \mathrm{v})$ pNPP was added to the supernatant in a 96-well plate (Corning Costar, NY, USA). The enzyme reaction was carried out at $37^{\circ} \mathrm{C}$ for 45 minutes and then stopped by the addition of a solution containing $2 \mathrm{M}$ $\mathrm{NaOH}$ (Merck, Germany) and $0.2 \mathrm{mM}$ EDTA (Sigma-Aldrich, MO, USA) in distilled water. The absorbance of pNP formed was read at $405 \mathrm{~nm}$ in a microplate reader (Synergy HT, BioTek Instruments, USA) and sample concentrations were read off from a standard curve made using pNP values ranging from 0 to $0.2 \mu \mathrm{mol} / \mathrm{mL}$. The obtained ALP concentrations were normalized against the DNA concentrations of the same samples. Samples without cells were used as control.

\subsubsection{Alizarin red staining and calcium deposition quantification}

Staining with alizarin red was performed in order to assess the matrix mineralization. Constructs were rinsed with PBS and fixed in 10\% (v/v) formalin (Sigma-Aldrich, Germany) for 1 hour. Samples were maintained in PBS until further staining with a $2 \%(\mathrm{w} / \mathrm{v})$ alizarin red solution (Merck, Germany) in ultrapure water, for 5 minutes, and finally washed with distilled water. Stained structures were visualized using a stereo microscope (Stemi 1000, Zeiss) and images captured by a digital camera (Canon PowerShot, G11).

The inorganic calcium quantification was performed using the o-cresolphtalein-complexon method with colorimetric detection (Roche Cobas kit, Roche Diagnostics, Germany), according to the manufacturer's instructions. Samples were incubated in a $6 \mathrm{M} \mathrm{HCl}$ solution (Analar Normapur, Germany) to remove and dissolve the calcium. In a 96-well plate (Corning Costar, NY, USA), $175 \mu \mathrm{L}$ of ethanolamine (1 M, pH 10.6) were mixed with $10 \mu \mathrm{L}$ of each sample and incubated for 5 minutes at RT. Then, $70 \mu \mathrm{L}$ of o-cresolphtalein-complexon (0.3 mM, hydroxy-8-quinoleine $13.8 \mathrm{mM}, \mathrm{HCl} 122 \mathrm{mM}$ ) were added and further incubated at $37^{\circ} \mathrm{C}$ for 2 minutes. The absorbance of the samples was read at $570 \mathrm{~nm}$ in a microplate reader. The calcium concentrations were extrapolated from the standard curve obtained using serial dilutions of a $200 \mathrm{mg} / \mathrm{L} \mathrm{CaCl} 2$ solution (Merck, Germany). Samples without cells were used as control.

\subsubsection{Immunodetection of bone-specific proteins}

Immunocytochemistry was performed using a mouse anti-human monoclonal antibody against osteocalcin (OCN; clone OC4-30, Abcam, Cambridge, UK; dilution 1:25), a rabbit anti-human polyclonal antibody against osteopontin (OPN; Abcam, Cambridge, UK; dilution 1:25), and a rabbit anti-human polyclonal antibody against collagen type I (Col I; Abcam, Cambridge, UK; dilution 1:50). Human ASCs grown both on the SF-PET and PET textile scaffolds were fixed in $10 \%(\mathrm{v} / \mathrm{v})$ formalin (Sigma-Aldrich, Germany) for 1 hour, and stored at $4 \stackrel{\circ}{ } \mathrm{C}$ in PBS until further use. Cells were permeabilized with $0.025 \%(v / v)$ of Triton X-100/PBS (Sigma-Aldrich, Portugal) and washed twice with PBS. To block unspecific reactions, samples were incubated with $3 \%(\mathrm{w} / \mathrm{v})$ bovine serum 
albumin solution (BSA; Sigma-Aldrich, Germany) in PBS for 45 minutes at RT, followed by incubation with the primary antibodies overnight at $4{ }^{\circ} \mathrm{C}$ in $1 \%(\mathrm{w} / \mathrm{v}) \mathrm{BSA} / \mathrm{PBS}$ solution. Samples were rinsed in PBS for 10 minutes and then incubated for 2 hours in the dark with the respective secondary fluorochrome-conjugated antibodies, anti-rabbit/mouse IgG (Invitrogen, Life Technologies, California, USA; dilution 1:100). Finally, samples were incubated for 15 minutes with DAPI solution (Sigma-Aldrich, Portugal; dilution 1:1000) for nuclei staining and then washed with PBS for further observation under a Zeiss LSM 510 Meta confocal laser scanning microscope.

\subsubsection{RNA isolation and real-time reverse transcriptase-polymerase chain reaction}

The expression of mRNA for the genes of interest was measured by real-time PCR analysis. Total RNA was extracted using RNeasy Mini Kit (Qiagen, Germany), following the manufacturer's instructions. After each culturing time, constructs were washed with PBS, immersed in $500 \mu \mathrm{L}$ of $\beta$-mercaptoethanol solution ( $\beta$-ME; Sigma-Aldrich, Portugal) in RLT buffer (dilution $1: 100)$ and stored at $-80^{\circ} \mathrm{C}$ until further use. Samples were thawed at RT and sonicated for 15 minutes at $20 \stackrel{\circ}{ } \mathrm{C}$ to ensure the complete lysing of the cells. After adding 500 $\mu \mathrm{L}$ of $70 \%(\mathrm{v} / \mathrm{v})$ ethanol solution to the lysates, total volumes were transferred to RNeasy spin columns placed in $2 \mathrm{~mL}$ collection tubes and centrifuged for 30 seconds at $8.0 \mathrm{rpm}$. Then, $700 \mu \mathrm{L}$ of RW1 buffer solution were added to the RNesay spin columns and centrifuged for 30 seconds at $8.0 \mathrm{rpm}$. The RNA pellets were washed twice with $500 \mu \mathrm{L}$ RPE buffer (RPE buffer was previous diluted with $70 \% \mathrm{v} / \mathrm{v}$ ethanol according to the instructions) and centrifuged for 30 seconds and then 2 minutes at $8.0 \mathrm{rpm}$. Finally, the total RNA pellets were reconstituted in $30 \mu \mathrm{L}$ of RNAse-free water (Gibco, Invitrogen, UK), added to the RNeasy spin columns and centrifuged for 1 minute at $8.0 \mathrm{rpm}$ into $1.5 \mathrm{~mL}$ collection tubes. RNA quantity and purity were assessed with a NanoDrop ND-1000 spectrophotometer (NanoDrop Technologies, USA). Samples with a 260/280 ratio between 1.6 and 2.0 were used for first-strand complementary DNA (cDNA) synthesis according to the protocol from the qScript $^{\mathrm{TM}}$ cDNA synthesis Kit (Quanta Biosciences, MD, USA) in a MiniOpticon real-time PCR detection system (BioRad, CA, USA). Briefly, a reaction mixture consisting of $4 \mu \mathrm{L}$ qScript Reaction Mix, $1 \mu \mathrm{L}$ qScript Reverse Transcriptase (RT), RNA template (100 ng total RNA) and nuclease-free water was prepared, in $20 \mu \mathrm{L}$ of final volume. The single-strand cDNA synthesis occurred by incubating the complete reaction mixture for 5 minutes at $22{ }^{\circ} \mathrm{C}$, followed by 30 minutes at $42{ }^{\circ} \mathrm{C}$ and terminated with an incubation of 5 minutes at $85{ }^{\circ} \mathrm{C}$. Amplification of the target cDNA for real-time PCR quantification was performed according to the manufacturer's instructions, using $1 \mu \mathrm{L} \mathrm{RT} \mathrm{cDNA} \mathrm{products,} 1 \mu \mathrm{M}$ each primer, $10 \mu \mathrm{L}$ PerfeCta SYBR Green FastMix (Quanta Biosciences, MD, USA) and nuclease-free water, in a final volume of $20 \mu \mathrm{L}$. The primers sequence, specific for each gene, namely glyceraldehyde-3-phosphate dehydrogenase (GAPDH), OCN, OPN and Col I are described in Table 1. 
Each real-time PCR run was carried out with an initial incubation at $95{ }^{\circ} \mathrm{C}$ for 2 minutes, followed by 45 cycles of denaturation (95 ${ }^{\circ}$, 10 seconds), annealing (specif ic for each gene, 25 seconds) and extension (72 ${ }^{\circ}, 30$ seconds) in a Mastercycler ${ }^{\circledR}$ ep realplex real-time PCR system (Eppendorf, USA). The transcripts expression data were normalized to the endogenous housekeeping gene glyceralde- hyde-3-phosphate-dehydrogenase (GAPDH) and the relative quantification was calculated using as calibrators the results obtained for each marker at day 7 in basal culture conditions, according to the Livak $\left(2^{-\Delta \Delta C T}\right)$ method [31].

Table 1: Primers list for the studied genes

\begin{tabular}{ccc}
\hline \multirow{2}{*}{ Gene } & \multicolumn{2}{c}{ Sequences } \\
\cline { 2 - 3 } & Torward (5'-3') & Reverse (5'-3') \\
\hline GAPDH & TGCACACCAACTGCTTAGC & GGCATGGACTGTGGTCATGAG \\
OCN & CTGGAGAGGAGCAGAACTGG & GGCAGCGAGGTAGTGAAGAG \\
OPN & TGAAACGAGTCAGCTGGATG & TGAAATTCATGGCTGTGGAA \\
Coll & AAGAACCCCAAGGACAAGAG & GTAGGTGATGTTCTGGGAGG \\
\hline
\end{tabular}

\subsection{In vivo angiogenesis evaluation}

\subsubsection{Chick chorioallantoic membrane assay}

CAM assay was performed according to the procedure described by Silva-Correia et al. [32] in a laminar-flow hood to minimize contamination. Briefly, white fertilized chicken eggs ( $n=120$; Pintobar, Portugal) were incubated at $37^{\circ} \mathrm{C}$ (Laboratory Incubator model B842 0; Termaks) for 3 days. A small hole was then created in the pointed end of the egg to allow dissociation of the CAM from the shell membrane. Additionally, a circular window was made into the egg shell, in order to evaluate embryo viability and access the CAM. Shell opening was then sealed with transparent tape $(\sim 50 \times 30 \mathrm{~mm}$. Staples $)$ to prevent dehydration and the eggs were returned to the incubator at $37 \stackrel{\circ}{\circ}$ until implantation of the SF-PET and PET textile scaffolds (diameter: 9 mm; height: $3 \mathrm{~mm}$ and $2.5 \mathrm{~mm}$, respectively).

Sterile textile scaffolds were implanted on the CAM at day 10 of embryonic development. Gelatin sponges with $9 \mathrm{~mm}$ diameter and $3 \mathrm{~mm}$ height were prepared from sterile Cutanplast ${ }^{\circledR}$ sponge (Mascia Brunelli S.p.a.) and used as negative control for angiogenesis. The shell windows were then protected with transparent tape and the eggs returned to the incubator at $37^{\circ} \mathrm{C}$ until day 14 of embryonic development. At the end of the assay, the embryos and their membranes were fixed in ovo by using freshly prepared paraformaldehyde (PFA; Merck) solution at $4 \%(\mathrm{v} / \mathrm{v})$ followed by incubation at $-80{ }^{\circ} \mathrm{C}$ for 10 minutes in an ultra-low freezer. The implanted specimens and the immediately adjacent CAM portions were cut and transferred to 6-well plates containing PFA 
solution at $4 \%(\mathrm{v} / \mathrm{v})$. Ex ovo images were then acquired using the AxioVision imaging software (release 4.8; Zeiss) connected to a AxioCAM ICc1 digital camera (Zeiss) attached to a stereomicroscope (Stemi 2000-C; Zeiss). The excised membranes were transferred to histological cassettes, embedded in paraffin, and cut into sections of $4 \mu \mathrm{m}$ thick using a microtome (Spencer 820, American Optical Company, NY, USA). Three independent CAM assays were performed and a minimum number of 18 specimens were used for each group.

\subsubsection{Analysis of blood vessel convergence}

A semi-quantitative method was used to evaluate the total number of macroscopic blood vessels converging toward the implanted textile scaffolds (SF-PET and PET) and gelatin sponges, as previously described [32]. The ex ovo images obtained at day 14 of embryonic development were analyzed by three independent observers after image processing using the WCIF ImageJ software program (US National Institutes of Health).

\subsubsection{Hematoxylin \& eosin staining}

The CAM sections were stained with H\&E (Sigma-Aldrich, Portugal) and observed under transmitted microscopy using an Axio Imager Z1m light microscope (Zeiss, Jena, Germany). Images were acquired using the Zen microscope processing software (Zeiss, Jena, Germany) connected to a digital camera AxioCam MRc5 (Zeiss, Jena, Germany).

\subsubsection{Immunohistochemical detection}

Representative CAM sections were subjected to immunohistochemical analysis using the streptavidin-biotin peroxidase complex kit (UltraVision Large Volume Detection System Anti-Polyvalent, HRP; Lab Vision, Thermo Scientific, CA, USA). After paraffin removal, sections were rehydrated and submitted to heat-induced antigen retrieval using $10 \mathrm{mM}$ citrate buffer $\left(\mathrm{pH} \mathrm{6}\right.$; Merck) for 20 minutes at $98^{\circ} \mathrm{C}$. To inactivate endogenous peroxidases, the samples were incubated in a $3 \%(\mathrm{v} / \mathrm{v})$ hydrogen peroxide solution $\left(\mathrm{H}_{2} \mathrm{O}_{2}\right.$; Panreac Química SAU) for 10 minutes, and then soaked in PBS. Sections were incubated in protein blocking solution (Ultra $V$ block; Lab Vision, Thermo Scientific, CA, USA) for 10 minutes followed by incubation with lectin (SNA-lectin; Vector Laboratories, Burlingame, CA, USA) for 1 hour at RT. Finally, sections were sequentially washed with PBS and incubated with the streptavidin-peroxidase complex for 10 minutes. A 3,3'-diamoonobenzidine solution (DAB; Vector Laboratories, Burlingame, USA) was used as chromogen. All sections were counterstained with Gill-2 hematoxylin (Merck, Germany). The histological sections were observed and images acquired as described in 2.5.3.

\subsection{Subcutaneous implantation}


The maintenance and use of animals were carried out in accordance to the Ethics Committee of University of Minho and approved by the Portuguese Licensing Authority (DGV-DSSPA). Six mice Hsd:ICR (CD-1) of 5 weeks old and average weight of 25-30 g (Harlan Laboratories, Sant Feliu de Codines, Spain) were used in this study. Each mouse was anesthetized by intraperitoneal injection of: ketamine (25 mg/kg) and medetomidine $(0.15 \mathrm{~mL} / \mathrm{kg})$ for anesthesia; cephalexin $(15 \mathrm{mg} / \mathrm{kg})$ as antibiotic; bupivacaine, pethidine $(5-10 \mathrm{mg} / \mathrm{kg})$ as analgesic. The hair of the mice was removed at the implantation area by shaving, followed by disinfection with $70 \%(\mathrm{v} / \mathrm{v})$ ethanol and iodine. In each mouse, four skin incisions (1 cm length) were made in the dorsal midline, two close from the head $(\mathrm{CH})$ and two far from the head $(\mathrm{FH})$. In the following, two sterile SF-PET textile scaffolds (diameter: $9 \mathrm{~mm}$; height: $3 \mathrm{~mm}$ ) and two sterile PET textile scaffolds (diameter: $9 \mathrm{~mm}$; height: $2.5 \mathrm{~mm}$ ) were implanted subcutaneously into the respective pocket and then the skin was sutured. Twelve specimens of both spacer fabrics were implanted. Fourteen and twenty eight days post-surgery, the mice were euthanized by injection of overdose pentobarbital sodium and the implanted materials together with the surrounded tissue retrieved. The explants were fixed with 10\% (v/v) formalin solution (Sigma-Aldrich, Germany) for at least 5 days at RT and then transferred to histological cassettes for immersion in paraffin, after dehydration through increasing series of ethanol concentrations (from 30\% up to $100 \% \mathrm{v} / \mathrm{v}$ ). Samples were then serially sectioned using a microtome (3.5 $\mu \mathrm{m}$ thick) for H\&E staining.

\subsection{Statistical analysis}

All the numerical results are presented as mean \pm standard deviation (SD). Statistical analysis was performed using the GraphPad Prism 5.0 (GraphPad Software, La Jolla, CA, USA). First, a Shapiro-Wilk test was used to ascertain about the data normality. The results from static mechanical properties were analyzed by means of the unpaired t-test using ten specimens for each group. For all the biological quantification assays, the differences between the experimental results were analyzed using a Kruskal-Wallis test followed by Dunn's multiple comparison test. Three independent experiments were performed for cell differentiation studies (ALP activity, calcium deposition quantification and real-time PCR analysis), and three samples were analyzed per group in each culturing time. A minimum number of 10 specimens were analyzed in each group for analysis of blood vessels convergence after CAM assay. The significance level was set to ${ }^{*} p<0.05,{ }^{* *} p<0.01,{ }^{* * *} p<$ 0.001 .

\section{Results}

\subsection{Morphological characterization}




\section{ACCEPTED MANUSCRIPT}

Figure 1 depicts the schematic drawings of the programmed knitted architectures to produce the SF-PET

(Figure 1a) and PET (Figure 1e) textile scaffolds, and provides details of the surface morphology and crosssections of the spacer scaffolds, obtained by SEM. The surface micrographs of SF-PET scaffolds (Figures $1 \mathrm{~b}$ and c) revealed a tight knit structure formed by natural silk filaments with regular size. Some loose filaments were observed in the SF matrices, which can be related to the physical wear resulting from the degumming process applied during sericin degumming. The PET-based external layers (Figures $1 \mathrm{f}$ and $\mathrm{g}$ ) showed filaments with the same size range of those present in the SF matrices, forming a loose knit structure. The cross-section micrographs of both SF-PET (Figure 1d) and PET (Figure 1h) spacer structures showed a significant threedimensionality introduced by the resilient monofilament of PET (z direction).

a

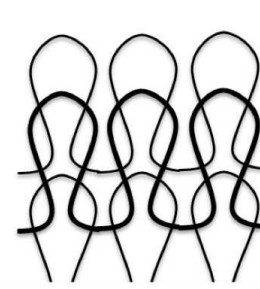

e

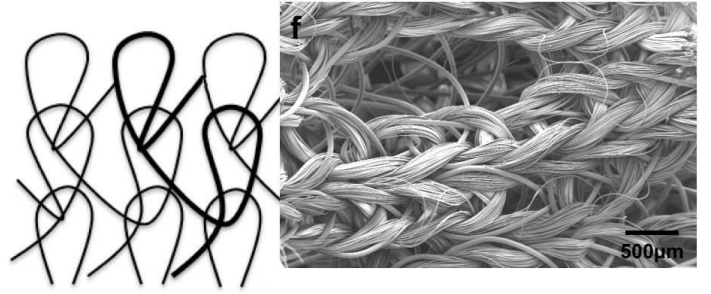

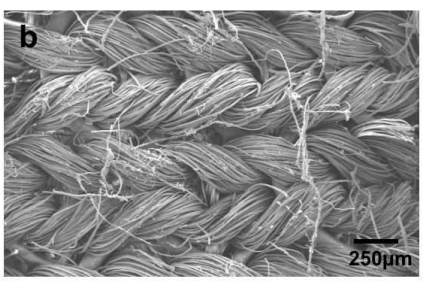
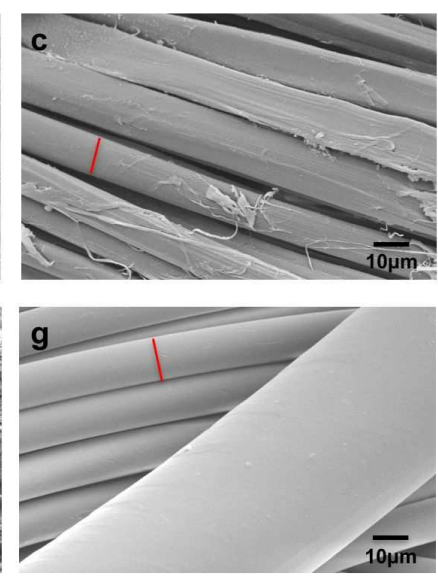
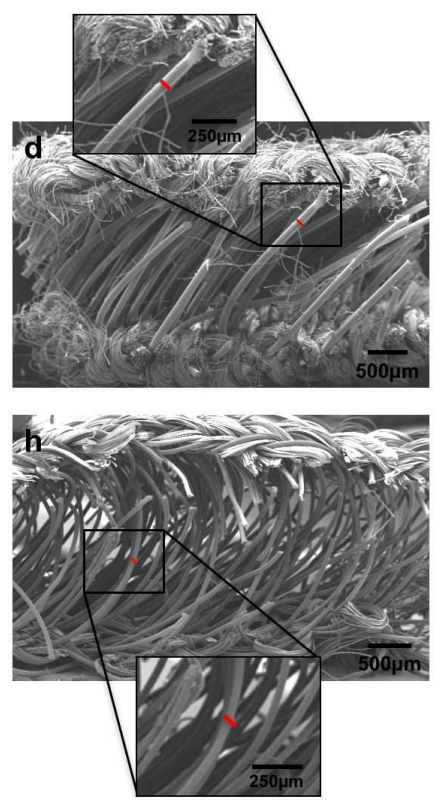

Figure 1. Illustrative images showing the wale and course components of the (a) weft and (e) warp knitting technologies, used for processing the SF and PET filaments in the scaffolds external layers, respectively. SEM micrographs of (b-d) SF-PET and (f-h) PET knitted spacer fabrics showing different levels of detail. Morphology of the (b, c) SF and (f, g) PET external layers from a top view perspective and the respective $(d, h)$ crosssections, showing the resilient PET monofilament (z direction). The red bars indicates: (c) SF and (g) PET fibres diameter $(\sim 15 \mu \mathrm{m})$; (d,h) PET monofilament diameter ( 100 $\mu \mathrm{m})$.

The qualitative and quantitative analysis of porosity, mean pore size and mean pore thickness of the spacer textile fabrics were assessed by micro-CT (Table 2). From the 3D reconstructions it was observed that both SFPET and PET spacer scaffolds presented high porosity and interconnected pores. Nevertheless, a higher porosity was observed at scaffolds' interior (Bulk), as compared to the external SF and PET layers (Top). MicroCT histomorphometric analysis also revealed that the external sheets of PET spacer scaffolds presented high 
porosity $(89.9 \pm 3.6 \%)$ and mean pore size $(355.6 \pm 3.4 \mu \mathrm{m})$, as compared to the porosity $(64.8 \pm 1.2 \%)$ and mean pore size $(179.5 \pm 5.0 \mu \mathrm{m})$ of SF matrices. No relevant differences for porosity were found among the scaffolds interior, in contrast to the mean pore size (1351.7 $\pm 16.7 \mu \mathrm{m}$ for PET, $587.6 \pm 4.6 \mu \mathrm{m}$ for SF-PET) and mean pore thickness $(125.9 \pm 1.7 \mu \mathrm{m}$ for PET, $75.3 \pm 1.3 \mu \mathrm{m}$ for SF-PET) that were found to be higher in the PET scaffolds interior. As the SF-PET scaffolds porosity increased, from the top to the bulk, the mean pore thickness tend to decrease. The same pattern was not observed for PET scaffolds, but the differences between the mean porosity at the scaffolds surface $(89.9 \pm 3.6 \%)$ and interior $(98.0 \pm 1.6 \%)$, were not so pronounced as in SF-PET scaffolds $(64.8 \pm 1.2 \%$ at surface, $95.0 \pm 1.1 \%$ at interior $)$.

Table 2. 3D reconstructions of the SF-PET and PET knitted spacer fabrics, mean porosity, pore size and trabeculae thickness, calculated from the micro-CT data.

\begin{tabular}{|c|c|c|c|c|}
\hline $\begin{array}{c}\text { Spacer textile } \\
\text { construct }\end{array}$ & 3D reconstruction & $\begin{array}{c}\text { Mean porosity } \\
(\%)\end{array}$ & $\begin{array}{c}\text { Mean pore size } \\
(\mu \mathrm{m})\end{array}$ & $\begin{array}{c}\text { Mean pore thickness } \\
(\mu \mathrm{m})\end{array}$ \\
\hline Top & & $64.8 \pm 1.2$ & $179.5 \pm 5.0$ & $115.4 \pm 3.7$ \\
\hline \multicolumn{5}{|l|}{ SF-PET } \\
\hline Bulk & & $95.0 \pm 1.1$ & $587.6 \pm 4.6$ & $75.3 \pm 1.3$ \\
\hline Top & & $89.9 \pm 3.6$ & $355.6 \pm 3.4$ & $108.2 \pm 2.1$ \\
\hline \multicolumn{5}{|l|}{ PET } \\
\hline Bulk & & $98.0 \pm 1.6$ & $1351.7 \pm 16.7$ & $125.9 \pm 1.7$ \\
\hline
\end{tabular}

\subsection{Mechanical properties}

Figure 2 shows the mechanical behavior of both SF-PET and PET spacer textile scaffolds evaluated under compression. The quasi-static compressive modulus (Figure 2a) determined in dry conditions was similar for both structures $(41.9 \pm 17.1 \mathrm{kPa}$ for SF-PET, $39.5 \pm 25.7 \mathrm{kPa}$ for PET). The same behavior was observed for the compressive strength of scaffolds, presented in the stress-strain plot (Figure 2b). The dynamic mechanical properties (wet conditions) of the SF-PET and PET spacer textile scaffolds assessed by DMA (Figure 2c) 
showed that the storage modulus ( $\left.E^{\prime}\right)$ of the SF-PET scaffolds increased at lower rates with increasing testing frequencies (from 0.1 to $10 \mathrm{~Hz}$ ), ranging from $39.1 \pm 8.7$ to $43.0 \pm 8.9 \mathrm{kPa}$. The $E$ ' of PET scaffolds presented no differences in all tested frequencies, ranging from $26.5 \pm 2.1$ to $27.6 \pm 2.5 \mathrm{kPa}$. In the frequency range tested, the storage modulus values obtained for SF-PET scaffolds were higher than for the PET scaffolds. Comparing the loss factor data, it was found that SF-PET spacer scaffolds presented higher loss factor values for the tested frequencies. Nevertheless, at higher frequencies (from $1 \mathrm{up}$ to $10 \mathrm{~Hz}$ ) the loss factor of SF-PET decreased. The tan $\delta$ of PET structures was constant in all tested frequencies.

a

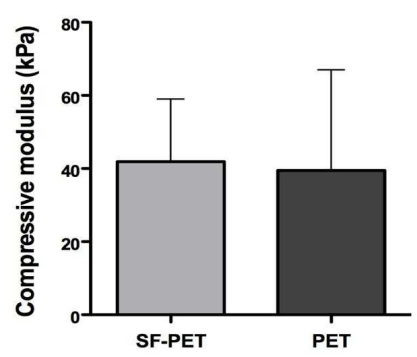

c

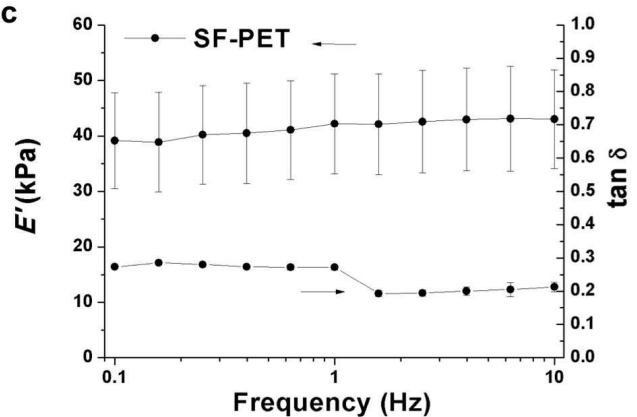

b
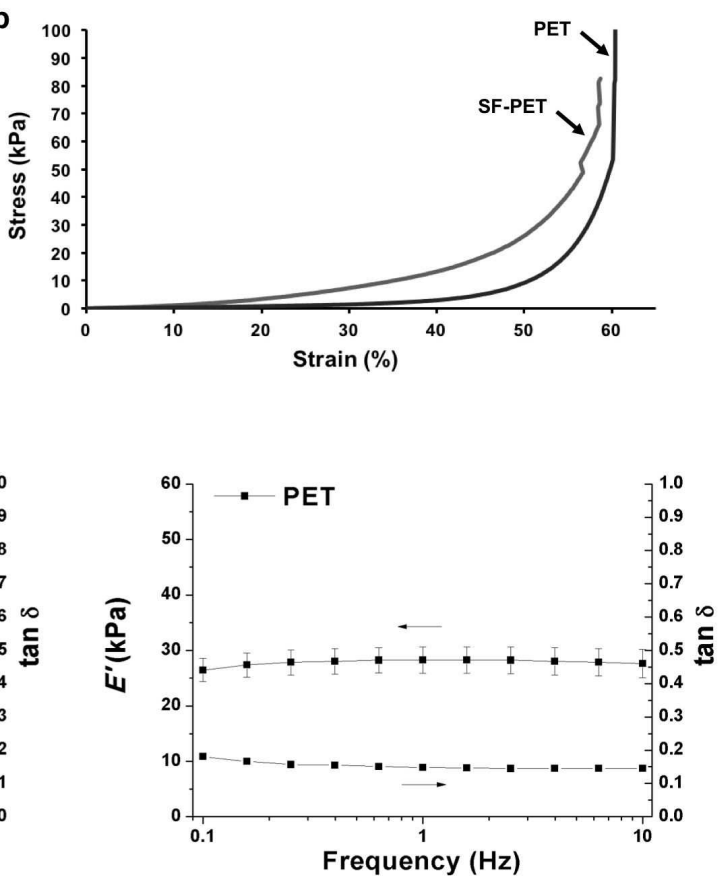

Figure 2. (a) Compressive modulus and (b) representative stress-strain plot of the SF-PET and PET knitted spacer fabrics measured in the dry state. (c) Storage modulus $(E)$ and loss factor (tan $\delta$ ) of the SF-PET and PET knitted spacer fabrics determined in the hydrated (phosphate-buffered saline solution, $37^{\circ} \mathrm{C}$ ) state, after 3 days of immersion.

\subsection{In vitro assessment of the textile-based scaffolds}

\subsubsection{Osteogenic differentiation in the textile-based scaffolds}

Quantitative analysis of ALP activity was determined up to 28 days of culturing (Figure 3). In osteogenic culture conditions, a significant increase of ALP activity was evidenced on the SF-PET constructs after 7 and 14 days of culture, in comparison to day $1\left({ }^{*} p<0.05\right.$ and ${ }^{* *} p<0.001$, respectively). From day 21 , significantly higher activity levels were also observed as compared to day $1\left({ }^{* *} p<0.01\right)$, however the ALP activity tend to decrease when compared to day 14 reaching stable values until day 28. PET constructs presented no significant 
differences in ALP activity until day 28, where significantly higher levels were detected as compared to day 1 $\left({ }^{\star * *} p<0.001\right)$ and day $\left.7{ }^{* \star} p<0.01\right)$. Comparing the results obtained for the different textile structures under the same osteogenic culture conditions, SF-PET constructs presented significantly higher activity levels from day 7 until day $28\left(a^{* \star *} p<0.001,{ }^{* *} p<0.01\right)$, as compared to PET. Concerning the cultures in basal medium, ALP activity significantly increased over the 28 days of culture $\left({ }^{* *} p<0.01 ;{ }^{* *} p<0.001\right)$ on the SF-PET textile structures, but the results obtained for PET structures revealed no significant differences until day 28 , as compared to day $\left.1{ }^{* \star *} \mathrm{p}<0.001\right)$. Also in basal culture conditions, SF-PET structures displayed a significantly higher ALP activity from day 14 until day $28\left(a^{\star \star \star} p<0.001\right)$ than PET constructs.

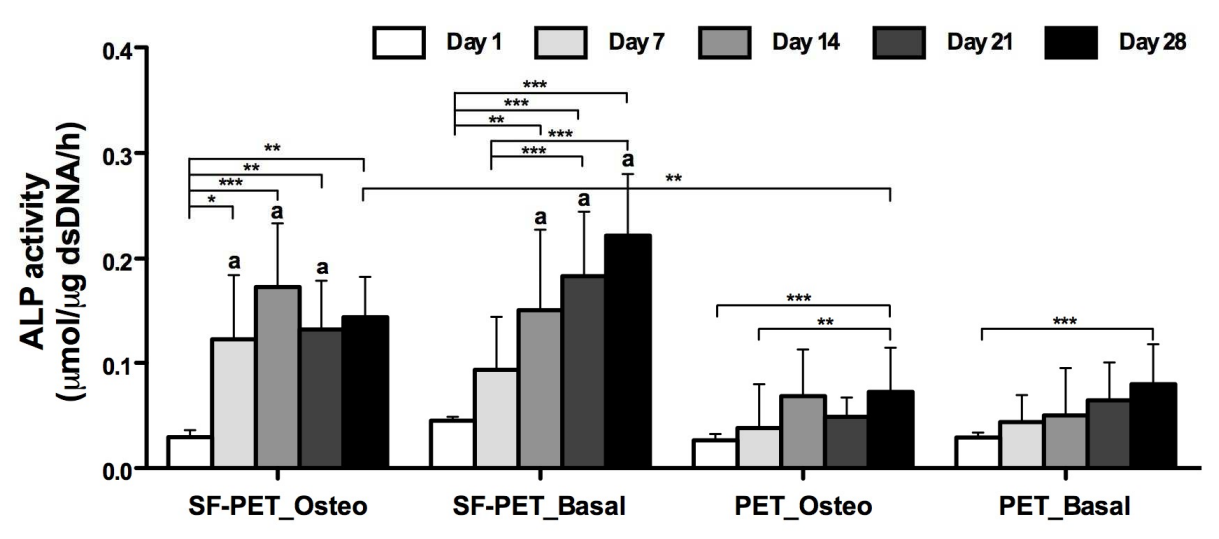

Figure 3. Normalized ALP activity of hASCs cultured on SF-PET and PET knitted spacer fabrics, after 1, 7, 14, 21 and 28 days in osteogenic and basal culture medium. ${ }^{a}$ Statistically significant when compared to the PET constructs at the same culture period and under the same culture conditions $\left({ }^{* * *} p<0.001\right)$. The exception is represented in the graphic.

\subsubsection{Extracellular matrix mineralization and elemental composition of the deposited matrix}

In figure $4 \mathrm{a}$, a significant increase in calcium concentration was observed after 21 days of hASCs culture in osteogenic differentiation medium on both SF-PET and PET constructs, as compared to day $7\left({ }^{* * *} p<0.001\right.$ and ${ }^{* *} p<0.01$, respectively $)$ and day $14\left({ }^{* *} p<0.01\right)$. After 28 days, significantly higher calcium concentration values were detected, as compared to day $7\left({ }^{* *} p<0.001\right)$ and day $14\left({ }^{* *} p<0.001\right)$ in both textile constructs. In basal culture conditions, only a residual calcium concentration was detected and no significant differences were observed for both scaffolds over the 28 days of cuture. In fact, the calcium concentration detected on SF-PET and PET constructs cultured in osteogenic conditions was significantly higher at day 21 and day $28\left({ }^{* * *} \mathrm{p}\right.$ 0.001), as compared to that obtained in basal conditions.

The ECM mineralization was confirmed by performing an alizarin red staining (Figure 4b). After 14 and 21 days of culture in osteogenic differentiation medium, evidences of ECM mineralization were observed on the SF-PET 
and PET constructs. The mineralization was more intense after 28 days of culture in both textile constructs, when compared to the first 21 days in the same osteogenic culture conditions. Moreover, it is visible that the stained areas are not homogeneously distributed within the scaffolds. When comparing the results obtained in osteogenic and basal culture conditions, a clear difference was observed. Both SF-PET and PET constructs showed a strong staining when cultured in osteogenic differentiation medium, while there were no evidences of ECM mineralization in basal culture conditions.

a

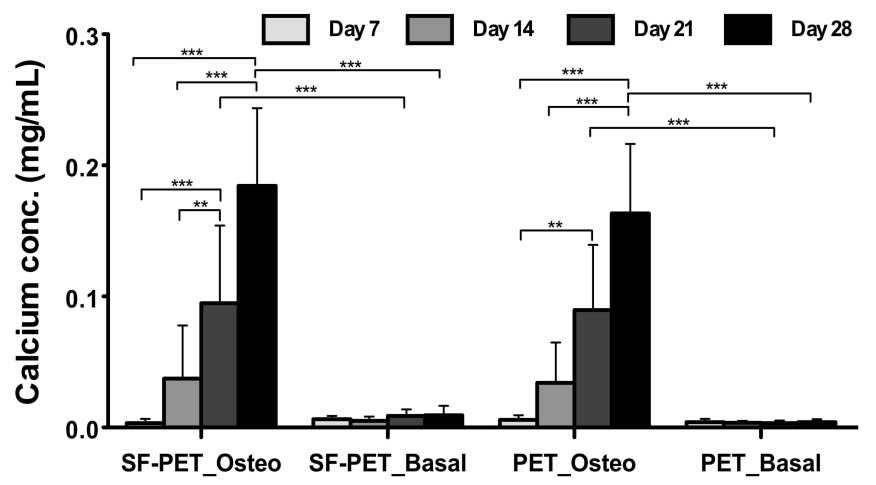

b

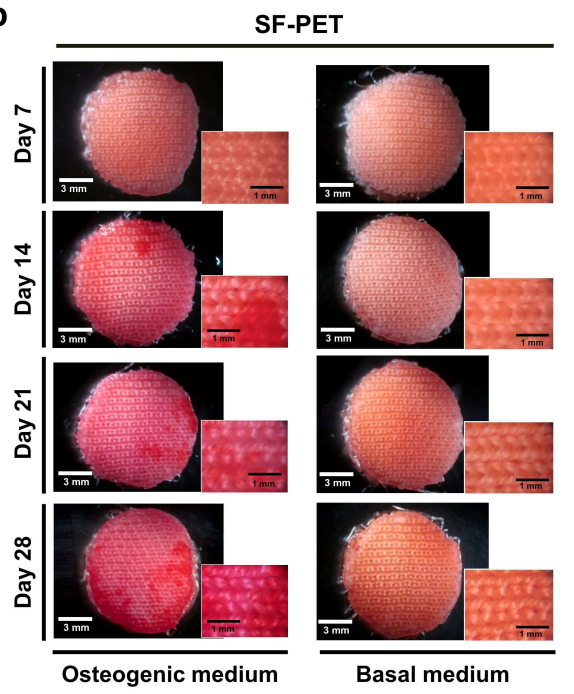

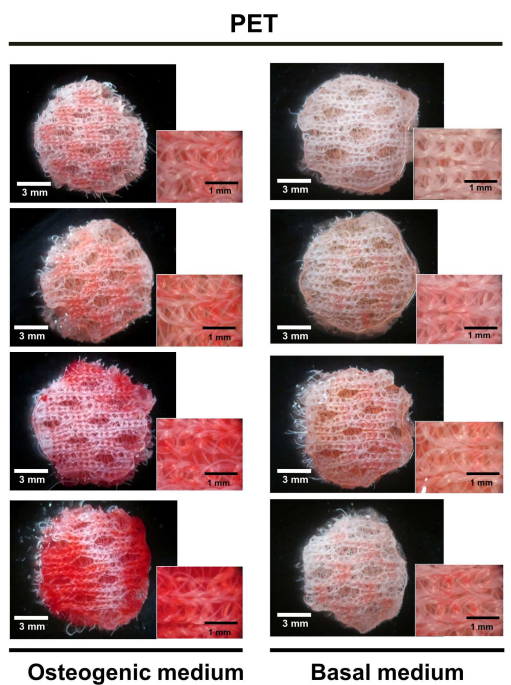

Osteogenic medium

Basal medium

Figure 4. (a) Calcium quantification on the SF-PET and PET knitted spacer fabrics, cultured with hASCs in osteogenic and basal medium for 7, 14, 21 and 28 days. (b) Alizarin red staining performed directly into the textile constructs, after 7, 14, 21 and 28 days of culture in osteogenic and basal medium.

The hASCs adhesion along the SF-PET and PET textile scaffolds was analyzed by SEM (supplementary Figure 1), showing that cells were able to attach, spread and deeply penetrate into the scaffolds' interior. From figure 5 , an increasing formation of mineralization nodules were observed on both SF-PET and PET constructs, from day 14 until day 28 in osteogenic culture conditions. Using EDS analysis it was possible to confirm that the nodules 
were composed mainly of calcium $(\mathrm{Ca})$ and phosphorous $(\mathrm{P})$, reaching $\mathrm{Ca} / \mathrm{P}$ ratio values between 1.65 to 1.79 on SF-PET constructs and 1,70 to 1.79 on PET contrcuts. Similar $\mathrm{Ca} / \mathrm{P}$ ratios were observed on both textile scaffolds at the different culture periods.
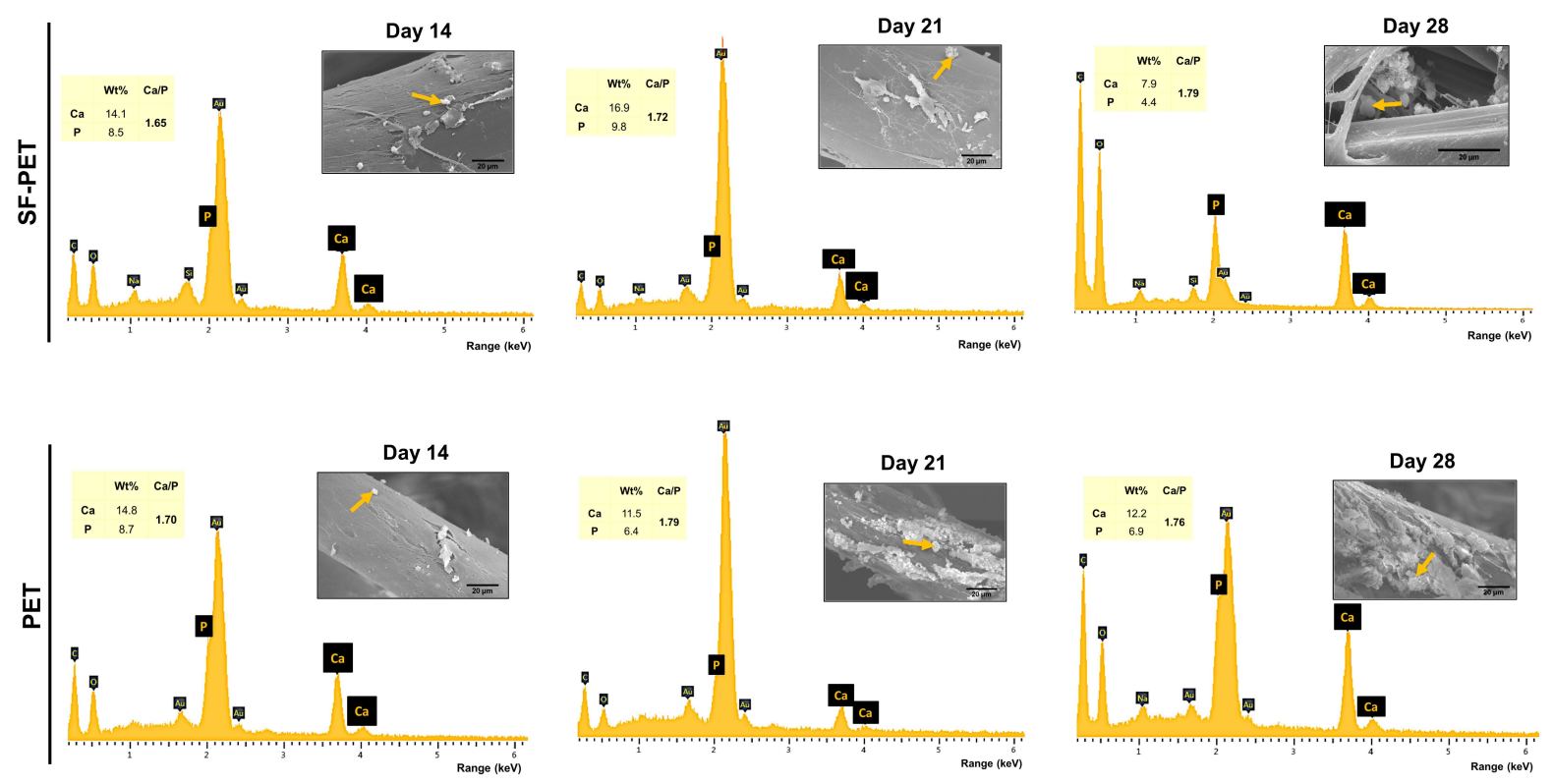

Figure 5. SEM micrographs (inset) and EDS spectra with $\mathrm{Ca} / \mathrm{P}$ ratios determined for the SF-PET and PET knitted spacer fabrics, after 14, 21 and 28 days of culture in osteogenic medium. The yellow arrows indicate mineralization nodules.

\subsubsection{Genotypic and phenotypic expression of osteogenic-related markers}

The differentiation of hASCs seeded onto the SF-PET and PET spacer textile scaffolds was analyzed by quantifying the mRNA expression of osteocalcin (OCN), osteopontin (OPN) and collagen type I (Col I) osteogenic markers (Figure 6). All mRNA transcripts were observed after 28 days in osteogenic and basal culture conditions (Figure 6a). The OCN and OPN gene expression presented no significant differences between the SF-PET and PET constructs cultured in osteogenic differentiation medium. In basal culture conditions, no mRNA expression was detected for these osteogenic-related markers both on SF-PET and PET constructs. For Col I gene expression, no significant differences were detected between the tested textile materials cultured under osteogenic and basal conditions. However, the mRNA expression of Col I was significantly higher in basal culture culture conditions, as compared to SF-PET $\left({ }^{*} \mathrm{p}<0.05\right)$ and PET $\left({ }^{* * *} \mathrm{p}<\right.$ 0.001 ) constructs cultured in osteogenic differentiation medium.

Complementary to the genotypic analysis, the osteogenic phenotype of hASCs seeded onto the SF-PET and PET spacer textile scaffolds was assessed by the immunodetection of OCN and OPN bone-specific 


\section{ACCEPTED MANUSCRIPT}

glycoproteins. The presence of Col I was also evaluated in order to determine the deposited collagenous matrix. Confocal micrographs (Figure 6b), indicated that in basal culture conditions only residual OCN and OPN were detected for hASCs cultured for 28 days on both SF-PET and PET textile scaffolds. However, in the presence of osteogenic differentiation medium, higher evidences of OCN and OPN were observed. In the case of Col I an intense protein expression was observed on SF-PET and PET constructs cultured both under osteogenic and basal culture conditions. Moreover, a uniformly distributed collagen deposition seemed to be detected on both textile scaffolds.

a

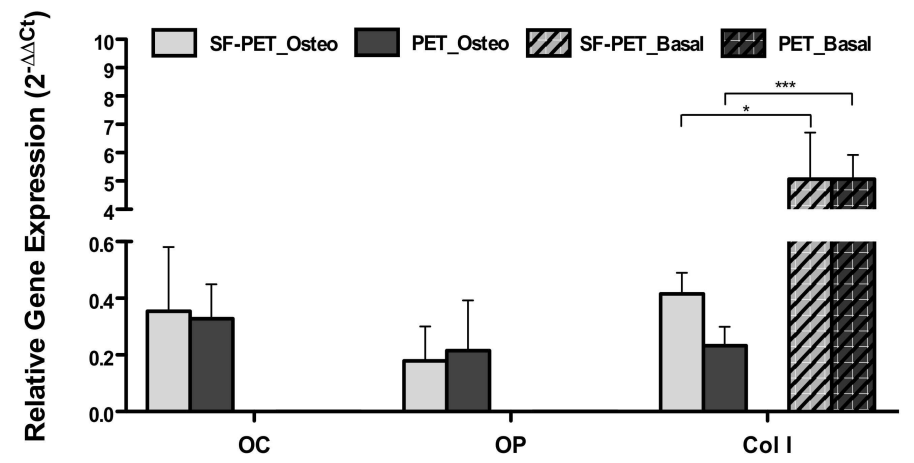

b
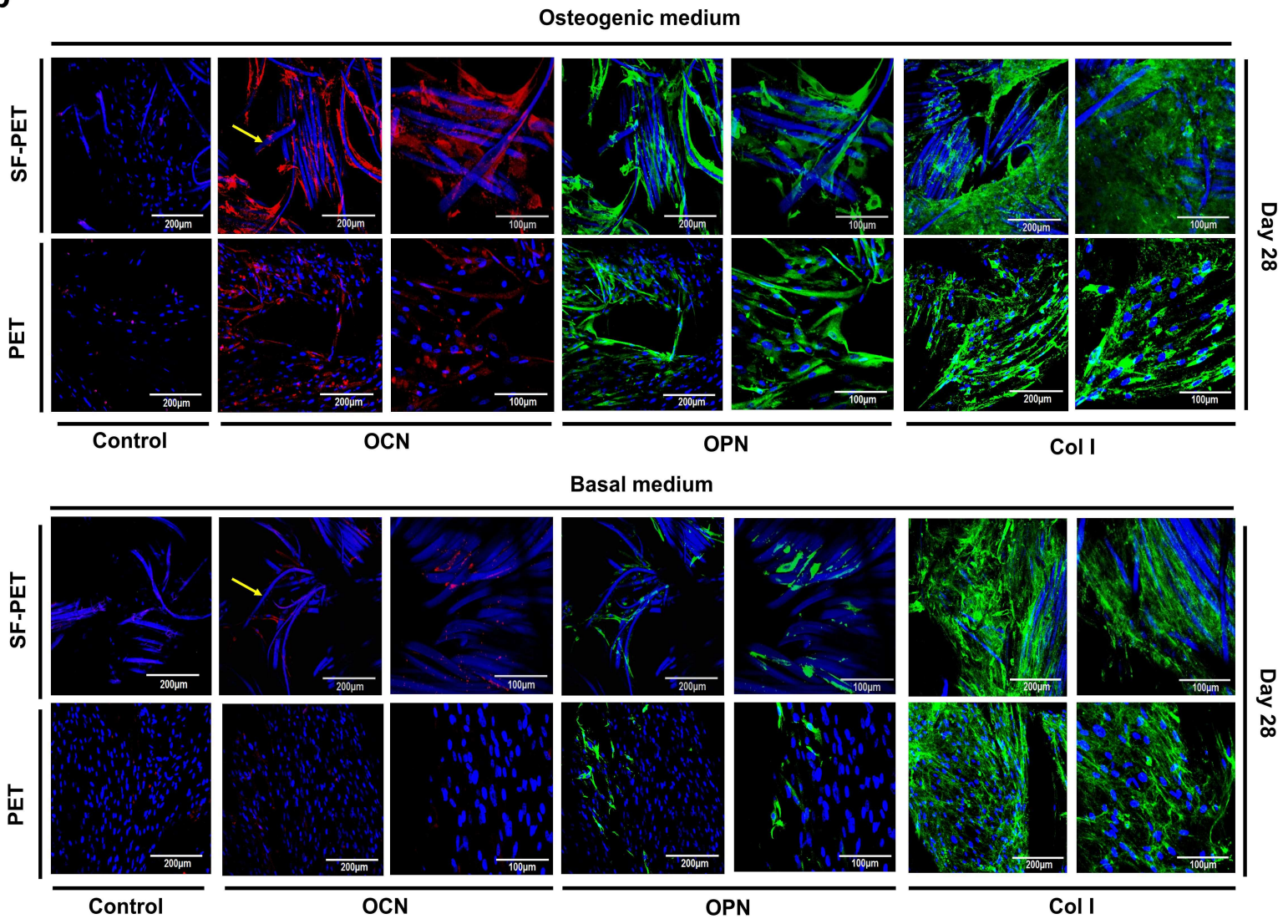
Figure 6. Quantitative and qualitative expression of osteogenic-related markers by hASCs after 28 days of culture on the SF-PET and PET knitted spacer fabrics. (a) RT-PCR results and (b) confocal micrographs of the detection of OCN, OPN and Col I in osteogenic and basal culture conditions. In representative immunofluorescence images nuclei are stained in blue, OCN in red, OPN and Col I in green. The yellow arrows indicate SF fibres stained in blue due to autofluorescence in the blue wavelength range. Scale bars: $200 \mu \mathrm{m}$ for low magnification images; $100 \mu \mathrm{m}$ for high magnification images.

\subsection{Angiogenic potential of the textile-based scaffolds}

The convergence of blood vessels toward the implanted structures was investigated ex ovo (Figure 7a). Macroscopically, there were no significant differences on the number of blood vessels converging to the PET textiles and control gelatin sponges. However, a significantly higher number of blood vessels converged to the SF-PET textiles, in comparison to the PET $\left({ }^{*} p<0.05\right)$ and gelatin sponges $\left({ }^{\star *} p<0.01\right)$. The stereomicroscopy photographs and H\&E staining images (Figure 7b) showed that both textile scaffolds were well integrated in the CAM after 4 days of implantation, allowing connective tissue infiltration. Complementarily, the immunohistochemical analysis of endothelial cells of chick origin performed with SNA-lectin (Figure 7b), revealed the presence of endothelial cells surrounding the textile fibres. Gelatin sponge implants were partially absorbed, as observed by the ex ovo and H\&E staining images. Immunohistochemistry showed the presence of endothelial cells contacting and infiltrating into the control gelatin sponges.
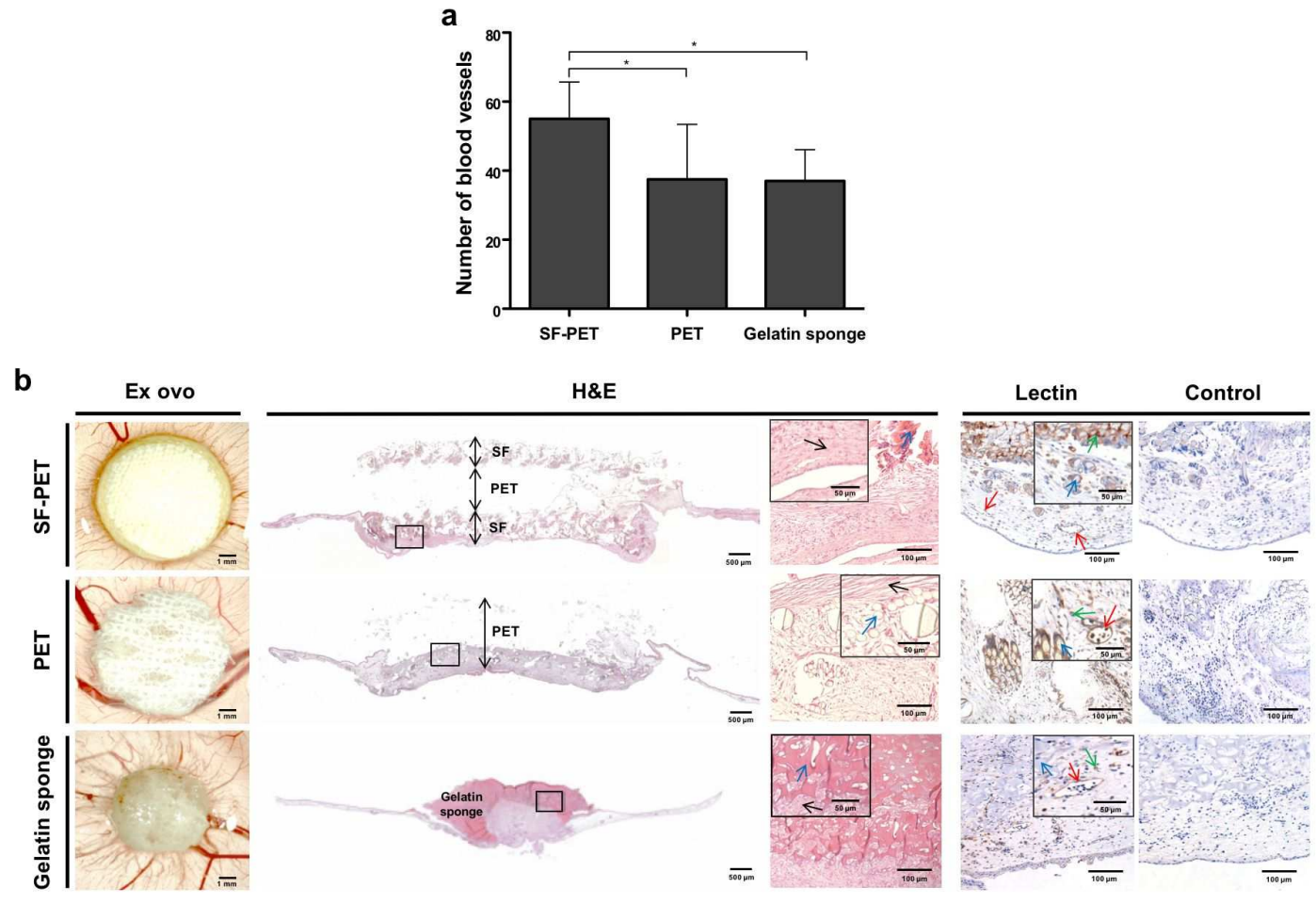
Figure 7. Chorioallantoic membrane (CAM) assay, after 4 days of SF-PET, PET textiles and gelatin sponges implantation. (a) Quantification of the macroscopic blood vessels converging towards the implanted structures. (b) Stereomicroscopy photographs of the explants and excised CAM sections of the implants stained with $\mathrm{H} \& \mathrm{E}$ and SNA-lectin. Squares represent the regions corresponding to the magnified stained images. The black arrows indicate connective tissue, the red arrows indicate blood vessels, the green arrows indicate infiltrated endothelial cells (brown) and the blue arrows indicate SF-PET, PET fibres or gelatin sponge.

\subsection{Subcutaneous implantation of the textile-based scaffolds}

The in vivo biological performance of the SF-PET and PET spacer textile scaffolds was assessed after subcutaneous implantation in mice. The macroscopic images of the explants showed that both SF-PET and PET scaffolds were integrated in the subcutaneous tissue, maintaining their integrity and shape after 14 (Figures8a and $b$ ) and 28 (Figures $8 g$ and $h$ ) days of implantation. The H\&E staining images showed the presence of connective tissue at the SF-PET (Figures $8 \mathrm{c}$ and j) and PET (Figures $8 \mathrm{e}$ and I) scaffolds surface and also infiltrating the scaffolds interior. It was also detected the presence of macrophages in the connective tissue, wrapping the fibres. Nevertheless, no signs of acute inflammation were observed, nor by the occurrence of edema after the implantation periods nor by the presence of neutrophils. Additionally, the infiltration of blood vessels was also identified on both textile structures (Figure 8d, f, I and k).

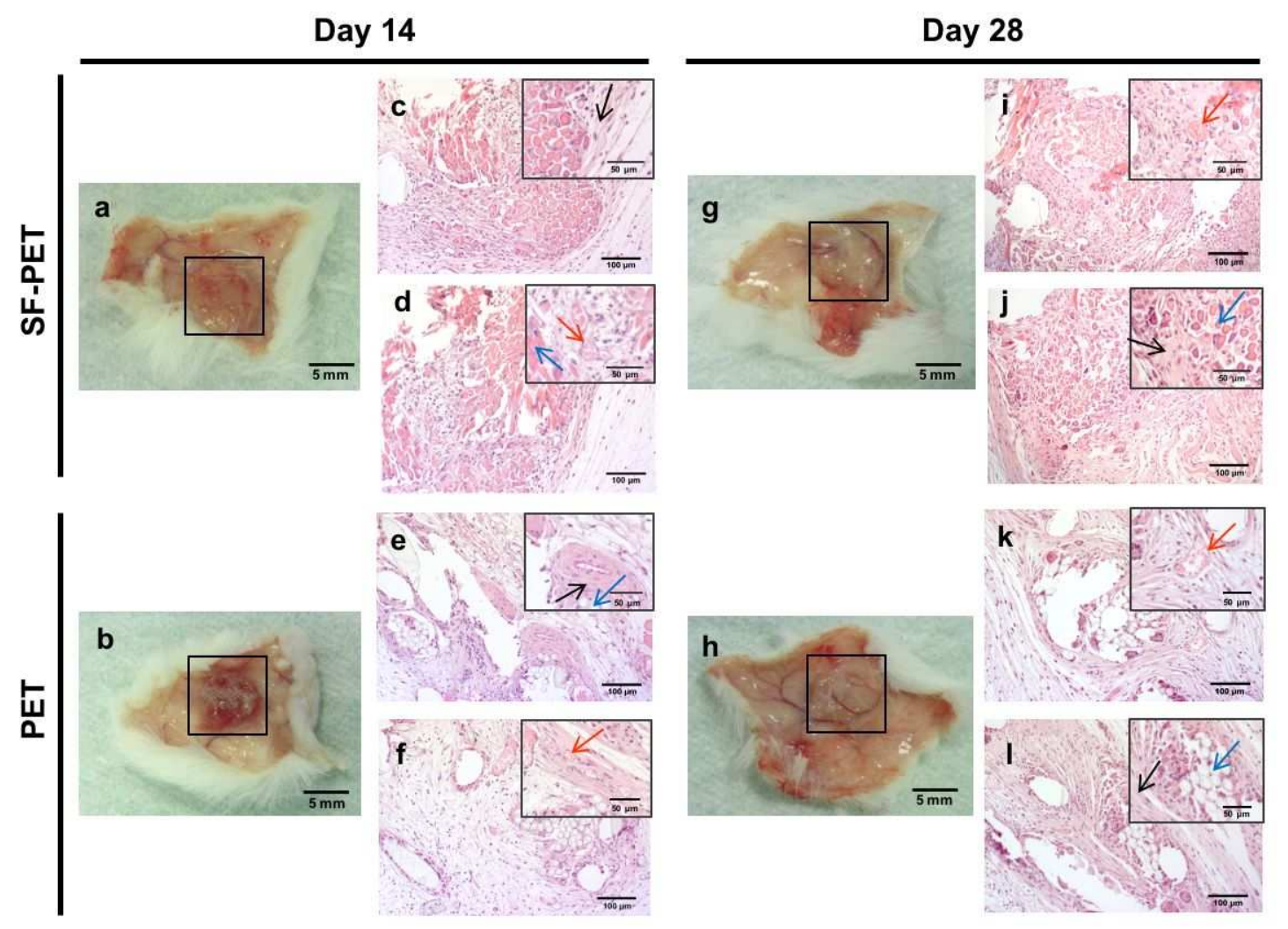


Figure 8. Subcutaneous implantation of the SF-PET and PET knitted spacer fabrics in mice. Macroscopic images of the explants after implantation for $(a, b) 14$ and $(g, h) 28$ days. H\&E staining of the SF (c, d, i, j) and PET $(e, f, k, l)$ explants. Squares represent the regions corresponding to the implanted SF and PET textile scaffolds. The black arrows indicate connective tissue, the red arrows indicate blood vessels and the blue arrows indicate SF-PET and PET fibres.

\section{Discussion}

Textile technologies are being increasingly adopted to generate highly precise and reproducible scaffolds for tissue engineering purposes. Different reports have proposed the use of knitting technologies as scaffolds for several TE applications, including tendons and ligaments [11], cartilage [33] or skin [34]. However, only few have proposed the use of these knitting technologies as a way to create architectures with sufficient mechanical properties according to the geometric parameters of the looms (yarn spacing and thickness) and fibre materials, for more targeted bone regeneration applications. For example, Kellomäki et al., [35] developed composite plates of weft-knitted PLA meshes attached on the surface of PLA films, showing that the composite structures were able to guide new bone formation and the mechanical properties of the composites were dependent on the mesh component. In previous studies by our group [14, 15, 36], standard weft-knitted SF and polybutylene succinate (PBS) scaffolds were proposed as a platform for the functional engineering of bone, showing that the knitting textile substrates exhibit better extensibility or compliance as compared to other textile structures, including the 3D woven structures previously proposed by Moutos et al. [9] as a possible strategy for cartilage TE applications. This technology has been applied for TE strategies that seek to restore the biomechanical functions of damaged musculoskeletal tissues $[9,37,38]$, particularly difficult to mimic in terms of biomechanical properties, as they are continualy subjected to complex loading patterns that require tissue arquitectures with preferably aligned fibre structures, as those present on knitting structures. Nevertheless, to fully recreate the necessary three-dimensionality and anisotropy found in bone tissue an increase in the three-dimensionality is required. To achieve this effect, in the herein developed scaffolds two external silk fabric layers are connected by a resilient PET monofilament ( $z$ direction) that not only increases the scaffolds' three-dimensionality but also replicates the anisotropy of flat bone [16]. Advanced materials can be reinforced by these textile plaftforms for structural applications. The control over fibre architecture is of potential interest for highly loaded structures, enabling fibres to be assembled in specific positions and with necessary orientations to optimize strength and stiffness locally [39]. The cellular components can also take advantage from this arquitecture by producing ECM to generate new tissue, while the scaffold material provides structural integrity and mechanical stability in the mean time [40]. In this study, the PET monofilament is mostly responsible for the resulting mechanical 
performance regardless of the composition of the external layers, as demonstrated by similar compressive modulus (Figure 2a) and strength (Figure 2b), presented by SF-PET and PET knitted spacer fabrics [41]. In a previous study, Walther et al. [13] observed that the compression strength of 3D textile scaffolds produced by electrostatic flocking changed depending on the fibre length that defined the scaffolds thickness, ranging from 10-36 kPa, where a higher fibre length resulted in lower compression strength. This was not the behavior presented by our samples, since the SF-PET scaffolds presented similar compressive modulus (Figure 2a) and strength (Figure 2b) to PET scaffolds, even presenting higher thickness. The anisotropic architecture of flat bone in all craniofacial region has a direct influence on its stiffness and strength. O'Mahony et al. [42] determined different compressive modulus on hydrated compact bones taken from an edentulous mandible, ranging from 114 to $907 \mathrm{MPa}$, depending on the tested direction. In another example, Misch et al. [43] observed that the ultimate compressive strength of different human mandibular trabecular bone regions in the absence of cortical plates, ranged from 1.7 to $5.38 \mathrm{MPa}$. This huge variability of available data is a consequence of testing bone in different directions, different regions and with diferent type of individuals. Therefore, the strength values obtained for the spacer scaffolds are difficult to compare with the above described values for native bone. Nevertheless, it is important to consider that bone tissue regeneration does not imply that the mechanical properties of the temporary bone substitute equal those of the natural bone. Scaffolds should present sufficient mechanical strength to support the hydrostatic pressures and to allow cells to recognize an adequate mechanical stimuli for ECM production, mineralization and reinforcement by native bone tissue. Different studies have demonstrated that a compressive modulus of only a few MPa are sufficient to meet all the requirements for an adequate bone regeneration $[44,45]$. In the present study we have designed a construct with great capacity to fold and to be easily adjusted and shaped to the bone defect, facilitating the surgical intervention. Interestingly when comparing the mechanical performance of the scaffolds in the dry state with the hydrated state (as in the real scenario), SF-PET presented higher storage modulus than the PET, as well as an increase of the storage modulus with the increase of the frequency, indicating a higher stiffness of this material. These results correlate well with the SEM (Figure 1) and micro-CT (Table 2) observations, where the SF-PET scaffolds presented lower porosity and mean pore size at the surface that might induce a higher stiffness of the structures in the hydrated state. The interaction of silk with water and its effect on the mechanical properties is well-known as reported previously by us $[14,15]$, and correlates well with the present study. The viscoelastic nature of both SF-PET and PET spacer scaffolds was also confirmed by the loss factor results (ranging from 0.2-0.3 and 0.150.2 , respectively), which means that the proposed scaffolds will be able to respond and recover from high 
physical loads before failing or breaking [46]. This is a very important concern considering that flat bones in the cranio-maxillofacial region are highly stimulated mechanically, due to the daily masticatory stresses that increase the activity in this area [5].

It is well recognized that mimic the anisotropy of bone in a scaffold can be a complex strategy for scaffold processing [47]. However, the flat bone in the maxillofacial region can constitute an interesting structure to recreate as it presents more regular architecture, which can be reproduced through 3D knitting technology. Up to now, the 3D textile technologies employed for TE purposes are limited to the use of synthetic monofilament fibres to cope with the stability of the fabrication process [33-35]. To our knowledge, this work is the first to report the use of a natural silk yarn to generate two weft-knitted SF layers spaced by a monofilament of PET as a medical textile. The resulting construct exhibits a thickness which can be adjusted to adequately fit the defect (Supplementary Figure 2). In this way we were able to generate a partially degradable porous construct which in the long term will fully integrate with bone. The mean pore size at these scaffolds interior, larger than $500 \mu \mathrm{m}$ (Table 2), has proved suitable for cell proliferation, nutrients exchange and capillaries formation, promoting rapid bone regeneration, correlating well with the literature [48]. Moreover, the presence of smaller pores in the outer layers, larger than $100 \mu \mathrm{m}$ (Table 2), is essential for the initial cell attachment [49]. SF-PET scaffolds presented higher fibre density than PET control scaffolds, which can be related to the different formed loops according to the applied knitting technology [50].

Human ASCs have emerged as a viable possibility for bone regeneration applications [20, 51]. Osteogenicinduced hASCs expressed the typical temporal sequence for ALP activity on SF-PET scaffolds, with a rise up until day 14 and a tendency to decrease at day 21 (Figure 3), accompanied by a significant increase in calcium content (Figure 4a). This behavior has been reported in other studies $[20,51]$ and is also in agreement with our results obtained from calcium deposition (Figure 4b), SEM and EDS analysis (Figure 5), showing the formation of a mineralized matrix with the presence of $\mathrm{Ca}$ and $\mathrm{P}$ elements. Moreover, in both textile constructs, the $\mathrm{Ca} / \mathrm{P}$ ratios (Figure 5) were very close to those typically found for the HA (1.67) and adult human bone $(1.71)[52,53]$. In a previous study by our group, Rodrigues et al., also demonstrated similar $\mathrm{Ca} / \mathrm{P}$ ratios after culturing fibrebased scaffolds with hASCs for 21 days in osteogenic culture conditions. From day 7 and day 21, significantly higher levels of ALP activity were presented by the SF-PET constructs cultured under osteogenic and basal conditions, respectively, as compared to PET constructs (Figure 3). These results can be explained by the lower number of cells quantified on the SF-PET constructs (data not shown) and the lower porosity and mean pore size observed on the external SF layers may also corroborates to this hypothesis (Table 2). Moreover, the 
sequencial increase of ECM mineralization observed at similar levels on SF-PET and PET constructs in osteogenic culture conditions (Figure 4), suggests that both knitted materials are potential matrices to support new bone deposition.

The genotypic (Figure 6a) and phenotypic (Figure 6b) analysis of the osteogenic-related markers OCN and OPN, showed the expression of both markers on SF-PET and PET constructs after 28 days of osteogenic culture, which may be indicative of the hASCs osteogenic differentiation [51]. Moreover, the non-expression of these osteogenic-related markers under basal culture conditions also support this hypothesis. Considering that OCN and OPN have a strong binding capacity to the inorganic component of bone ECM [7], these results also corroborate to our ECM mineralization observations (Figure 4 and 5). The relative gene expression of Col I was significantly higher in basal culture conditions for both textile constructs, which may also be related to the ostegenic differentiation of hASCs in osteogenic culture conditions, accompanied by a continuous deposition of the collagen matrix in basal conditions [54].

All together, the obtained results demonstrated the effectiveness of the proposed textile-based scaffolds on the differentiation of hASCs into osteoblasts and in the deposition of mineralized ECM. In a previous study by our group, Correia et al., [20] showed the ability of silk-based scaffolds to support hASCs osteogenic differentiation, confirmed by the typical course of ALP activity, calcium deposition and immunostaining of osteogenic-related markers. Mandal et al., [22] have also shown that micron-sized silk fibres used as reinforcement in a compact fibre composite favored the differentiation of BMSCs toward bone-like cells, based on biochemical and gene expression of bone markers. Walther et al., [13] presented novel 3D scaffolds generated by a flock textile technology that allowed hMSCs attachment, proliferation and differentiation along the osteoblastic lineage, revealing that the fibres' orientation and the highly porous structure had a positive influence over cell response and resulting biological mechanisms. Thus, the pre-establishment of the fibres' alignment on the development of textile-based scaffolds represents an important concert, since it can directly influence the differentiation potential of cells over the structures.

The quantification of convergent macroscopic blood vessels on CAM assay, demonstrated that SF-PET textile structures presented a significantly higher angiogenic capacity, as compared to PET textiles and gelatin sponges (Figure 7a). As a natural protein fibre, SF presents not only controlled degradability [14], as superior in vivo compatibility and angiogenic potential [55]. This is in agreement to the obtained histological results where SF-PET scaffolds presented good integration in the CAM (Figure 7b). These features are very important when considering a scaffold to be used as implant material. It should be well integrated in the first stage of 
implantation, but continuouslly absorbed to replace the new-formed tissue [32]. In fact, the chemical stability of the synthetic polymer PET [56] and the fact that gelatin sponges are often used in the CAM assay without influencing angiogenesis [32], also contribute to evidenciate the potential of SF in the present application. Both studied scaffolds allowed the infiltration of endothelial cells and the formation of microscopic blood vessels (Figure 7b), showing their potential as angiogenesis carriers. These textile constructs also showed good integrity and dimensional stability for the studied period of subcutaneous implantation (14-28 days) (Figure 8). This was expected for PET constructs [57] but in case of SF no signs of degradation were visible. In a previous study by our group [14] the degradability of SF knitted scaffolds (single layer) was studied using a solution of PBS containing $3.5 \mathrm{U} / \mathrm{mL}$ of protease XIV. These in vitro results present a weight loss of $5 \%$ after 5 days, which correlates well with the present study, indicating a slow proteolitically degradation of native SF fibres mostly due to its high crystalline content ( $\beta$-sheet conformation). In a previous study, Dal Pra et al., [58] observed that nonwoven SF cords in $\beta$-sheet form presented by day 180 of mice implantation a non-appreciable biodegradation, which corroborates the results obtained in our study. These results also indicate that scaffolds are able to retain good mechanical properties in vivo, which is in agreement with the wet compressive modulus analysis. The observation of a thick layer of connective tissue involving the fibres and infiltrating the scaffolds was found on both textile constructs, as well as the presence of vessels formed inside the scaffolds. The high porosity and fibre alignement in the structures may have contributed for these results [40]. It has been reported that fibre-like networks can have a positive influence over cell behavior and in vivo implantation, as it resembles the fibrous architecture of the natural ECM environment [8]. For example, Sanders et al., [59] have demonstrated that the subcutaneous implantation of textile scaffolds in mice resulted in different tissue thicknesses surrounding the structures, depending on the fibres' diameters. The results obtained for PET constructs can also evidence the positive influence of the proposed fibre-based networks with respect to in vitro and in vivo response. Unlike silk, whose surface chemistry has shown to positively influence cell behavior in different scaffolding strategies [14], the chemical stability of PET is not expected to induce any biological effects, which indicates that the cell viability, proliferation and osteogenic differentiation on the PET scaffolds and their in vivo performance can be related to the architecture and morphological features of the construct, as well as to the high molecular orientation on PET fibres due to shear stress during processing [60]. Besides of the promising results presented herein, the possibility of having an automated production of the proposed biotextiles represents great prospects for their possible industrialization and application in tissue engineering. We have addressed flat bone tissue 
engineering for modelling the presented scaffolds, but other specific applications can be considered due to the versatility of process.

\section{Conclusions}

In this study, novel 3D biotextile architectures composed of knitted SF and PET fibres spaced by a monofilament of PET, are proposed for the regeneration of bone tissue and a possible strategy for craniofacial bone scaffolding. The scaffolds presented suitable porosity and mechanical properties for bone applications, with special relevance for the superior mechanical properties of SF-PET textile scaffolds in the hydrated state. The in vitro osteogenic differentiation of the seeded hASCs was represented on SF-PET scaffolds by the peak of ALP activity after 14 days of osteogenic culture and subsequent deposition of mineralized ECM, also detected on PET constructs. Additionally, the genotypic expression and immunodetection of bone-specific transcripts also confirmed the osteogenic differentiation of hASCs cultured on SF-PET and PET spacer contructs. The in vivo studies revealed that SF-PET scaffolds induced higher angiogenic effect when performing a CAM assay and both textile structures allowed tissue ingrowth's when subcutaneously implanted in mice, with minimum foreign body reaction. Although a complementary in vivo study is necessary to evaluate the long-term performance and stability of the scaffolds in craniofacial bone defects, the obtained results indicate that the developed 3D biotextiles are promising structures for bone regeneration applications. Moreover, the structural adaptability of the knitted spacer fabrics increase the scaffolds applicability for different regenerative strategies, including for bone regeneration at the craniofacial complex.

\section{Acknowledgments}

Viviana Ribeiro was awarded a PhD scholarship (PD/BD/113806/2015) under the financial support from FCT/MCTES (Fundação para a Ciência e a Tecnologia/ Ministério da Ciência, Tecnologia, e Ensino Superior) and FSE/POCH (Fundo Social Europeu através do Programa Operacional do Capital Humano), PD/59/2013. The authors are greatful for the support from FCT through the Tissue2Tissue Project (PTDC/CTM/105703/2008). The FCT distinction attributed to J.M. Oliveira and A.L. Oliveira under the Investigator FCT program IF/00423/2012 and IF/00411/2013, respectively, are also greatly acknowledged. The authors are grateful to Teresa Oliveira for the assistance with the immunohistochemical staining and to Raquel Maia for the assistance at the cell culture laboratory. 


\section{References}

[1] M. Miura, Y. Miura, W. Sonoyama, T. Yamaza, S. Gronthos, S. Shi, Bone marrow - derived mesenchymal stem cells for regenerative medicine in craniofacial region, Oral diseases 12(6) (2006) 514-522.

[2] V. Petrovic, P. Zivkovic, D. Petrovic, V. Stefanovic, Craniofacial bone tissue engineering, Oral Surgery, Oral Medicine, Oral Pathology and Oral Radiology 114(3) (2012) e1-e9.

[3] H. Schöning, R. Emshoff, Primary temporary AO plate reconstruction of the mandible, Oral Surgery, Oral Medicine, Oral Pathology, Oral Radiology, and Endodontology 86(6) (1998) 667-672.

[4] Y. Kinoshita, H. Maeda, Recent developments of functional scaffolds for craniomaxillofacial bone tissue engineering applications, The Scientific World Journal 2013 (2013).

[5] B. Ward, S. Brown, P. Krebsbach, Bioengineering strategies for regeneration of craniofacial bone: a review of emerging technologies, Oral diseases 16(8) (2010) 709-716.

[6] F. Yang, R. Murugan, S. Wang, S. Ramakrishna, Electrospinning of nano/micro scale poly (L-lactic acid) aligned fibers and their potential in neural tissue engineering, Biomaterials 26(15) (2005) 2603-2610.

[7] A. Martins, M.L.A. da Silva, S. Faria, A.P. Marques, R.L. Reis, N.M. Neves, The influence of patterned nanofiber meshes on human mesenchymal stem cell osteogenesis, Macromolecular Bioscience 11(7) (2011) 978-987.

[8] R. Sumanasinghe, M.W. King, The applications of biotextiles in tissue engineering, Research Journal of Textile and Apparel 9(3) (2005) 80-90.

[9] F.T. Moutos, L.E. Freed, F. Guilak, A biomimetic three-dimensional woven composite scaffold for functional tissue engineering of cartilage, Nature materials 6(2) (2007) 162-167.

[10] H. Liu, H. Fan, Y. Wang, S.L. Toh, J.C. Goh, The interaction between a combined knitted silk scaffold and microporous silk sponge with human mesenchymal stem cells for ligament tissue engineering, Biomaterials 29(6) (2008) 662-674.

[11] X. Chen, Y.Y. Qi, L.L. Wang, Z. Yin, G.L. Yin, X.H. Zou, H.W. Ouyang, Ligament regeneration using a knitted silk scaffold combined with collagen matrix, Biomaterials 29(27) (2008) 3683-3692.

[12] C. Heinemann, S. Heinemann, A. Bernhardt, H. Worch, T. Hanke, Novel textile chitosan scaffolds promote spreading, proliferation, and differentiation of osteoblasts, Biomacromolecules 9(10) (2008) 2913-2920.

[13] A. Walther, B. Hoyer, A. Springer, B. Mrozik, T. Hanke, C. Cherif, W. Pompe, M. Gelinsky, Novel textile scaffolds generated by flock technology for tissue engineering of bone and cartilage, Materials 5(3) (2012) 540-557.

[14] L.R. Almeida, A.R. Martins, E.M. Fernandes, M.B. Oliveira, V.M. Correlo, I. Pashkuleva, A.P. Marques, A.S. Ribeiro, N.F. Durães, C.J. Silva, New biotextiles for tissue engineering: development, characterization and in vitro cellular viability, Acta Biomaterialia 9(9) (2013) 8167-8181.

[15] V.P. Ribeiro, L.R. Almeida, A.R. Martins, I. Pashkuleva, A.P. Marques, A.S. Ribeiro, C.J. Silva, G. Bonifácio, R.A. Sousa, R.L. Reis, Influence of different surface modification treatments on silk biotextiles for tissue engineering applications, Journal of Biomedical Materials Research Part B: Applied Biomaterials (2015).

[16] A. Davies, J. Williams, The use of spacer fabrics for absorbent medical applications, Journal of Fiber Bioengineering and Informatics 1(4) (2009) 321330.

[17] C. Chua, K. Leong, K. Tan, F. Wiria, C. Cheah, Development of tissue scaffolds using selective laser sintering of polyvinyl alcohol/hydroxyapatite biocomposite for craniofacial and joint defects, Journal of Materials Science: Materials in Medicine 15(10) (2004) 1113-1121.

[18] J.L. Moreau, H.H. Xu, Mesenchymal stem cell proliferation and differentiation on an injectable calcium phosphate-chitosan composite scaffold, Biomaterials 30(14) (2009) 2675-2682.

[19] Z.S. Haidar, R.C. Hamdy, M. Tabrizian, Delivery of recombinant bone morphogenetic proteins for bone regeneration and repair. Part B: Delivery systems for BMPs in orthopaedic and craniofacial tissue engineering, Biotechnology letters 31(12) (2009) $1825-1835$.

[20] C. Correia, S. Bhumiratana, L.P. Yan, A.L. Oliveira, J.M. Gimble, D. Rockwood, D.L. Kaplan, R.A. Sousa, R.L. Reis, G. Vunjak-Novakovic, Development of silk-based scaffolds for tissue engineering of bone from human adipose-derived stem cells, Acta Biomaterialia 8(7) (2012) $2483-2492$.

[21] L. Meinel, R. Fajardo, S. Hofmann, R. Langer, J. Chen, B. Snyder, G. Vunjak-Novakovic, D. Kaplan, Silk implants for the healing of critical size bone defects, Bone 37(5) (2005) 688-698.

[22] B.B. Mandal, A. Grinberg, E.S. Gil, B. Panilaitis, D.L. Kaplan, High-strength silk protein scaffolds for bone repair, Proceedings of the National Academy of Sciences 109(20) (2012) 7699-7704.

[23] K.H. Kim, L. Jeong, H.N. Park, S.Y. Shin, W.H. Park, S.C. Lee, T.I. Kim, Y.J. Park, Y.J. Seol, Y.M. Lee, Y. Ku, I.C. Rhyu, S.B. Han, C.P. Chung, Biological efficacy of silk fibroin nanofiber membranes for guided bone regeneration, Journal of Biotechnology 120(3) (2005) 327-339.

[24] Q. Han, P. Yang, Y. Wu, s. Meng, L. Sui, L. Zhang, L. Yu, Y. Tang, H. Jiang, D. Xuan, Epigenetically Modified Bone Marrow Stromal Cells (BMSCs) in Silk Scaffolds Promote Craniofacial Bone Repair and Wound Healing, Tissue Engineering (ja) (2015).

[25] A. Woloszyk, S.H. Dircksen, N. Bostanci, R. Müller, S. Hofmann, T.A. Mitsiadis, Influence of the mechanical environment on the engineering of mineralised tissues using human dental pulp stem cells and silk fibroin scaffolds, (2014).

[26] B. Levi, M.T. Longaker, Concise Review: Adipose - Derived Stromal Cells for Skeletal Regenerative Medicine, Stem cells 29(4) (2011) 576-582.

[27] M.T. Cerqueira, R.P. Pirraco, T.C. Santos, D.B. Rodrigues, A.M. Frias, A.R. Martins, R.L. Reis, A.P. Marques, Human adipose stem cells cell sheet constructs impact epidermal morphogenesis in full-thickness excisional wounds, Biomacromolecules 14(11) (2013) $3997-4008$.

[28] J.C. Bodle, A.D. Hanson, E.G. Loboa, Adipose-derived stem cells in functional bone tissue engineering: lessons from bone mechanobiology, Tissue Engineering Part B: Reviews 17(3) (2011) 195-211.

[29] Y.-D.C. Halvorsen, D. Franklin, A.L. Bond, D.C. Hitt, C. Auchter, A.L. Boskey, E.P. Paschalis, W.O. Wilkison, J.M. Gimble, Extracellular matrix mineralization and osteoblast gene expression by human adipose tissue-derived stromal cells, Tissue engineering 7(6) (2001) 729-741.

[30] S.M. Mihaila, A.K. Gaharwar, R.L. Reis, A. Khademhosseini, A.P. Marques, M.E. Gomes, The osteogenic differentiation of SSEA-4 sub-population of human adipose derived stem cells using silicate nanoplatelets, Biomaterials 35(33) (2014) 9087-9099.

[31] K.J. Livak, T.D. Schmittgen, Analysis of relative gene expression data using real-time quantitative PCR and the 2(T)(-Delta Delta C) method, Methods 25(4) (2001) 402-408.

[32] J. Silva-Correia, V. Miranda-Gonçalves, A.J. Salgado, N. Sousa, J.M. Oliveira, R.M. Reis, R.L. Reis, Angiogenic potential of gellan-gum-based hydrogels for application in nucleus pulposus regeneration: in vivo study, Tissue Engineering Part A 18(11-12) (2012) 1203-1212.

[33] G. Chen, T. Sato, T. Ushida, R. Hirochika, Y. Shirasaki, N. Ochiai, T. Tateishi, The use of a novel PLGA fiber/collagen composite web as a scaffold for engineering of articular cartilage tissue with adjustable thickness, Journal of Biomedical Materials Research Part A 67(4) (2003) 1170-1180.

[34] K.W. Ng, J. Louis, T. Ho, B. Saey, H.N. Achuth, J. Lu, S. Moochhala, T.C. Lim, D.W. Hutmacher, Characterization of a novel bioactive poly [(lactic acid)co-(glycolic acid)] and collagen hybrid matrix for dermal regeneration, Polymer international 54(10) (2005) 1449-1457. 
[35] M. Kellomäki, H. Niiranen, K. Puumanen, N. Ashammakhi, T. Waris, P. Törmälä, Bioabsorbable scaffolds for guided bone regeneration and generation, Biomaterials 21(24) (2000) 2495-2505.

[36] V.P. Ribeiro, L.R. Almeida, A.R. Martins, I. Pashkuleva, A.P. Marques, A.S. Ribeiro, C.J. Silva, G. Bonifácio, R.A. Sousa, A.L. Oliveira, Modulating cell adhesion to polybutylene succinate biotextile constructs for tissue engineering applications, Journal of Tissue Engineering and Regenerative Medicine (2016).

[37] F.T. Moutos, F. Guilak, Functional properties of cell-seeded three-dimensionally woven poly(epsilon-caprolactone) scaffolds for cartilage tissue engineering, Tissue Engineering Part A 16(4) (2010) 1291-1301.

[38] Z. Ge, F. Yang, J.C. Goh, S. Ramakrishna, E.H. Lee, Biomaterials and scaffolds for ligament tissue engineering, Journal of biomedical materials research Part A 77(3) (2006) 639-652.

[39] U. Wollina, M. Heide, W. Muller-Litz, D. Obenauf, J. Ash, Functional textiles in prevention of chronic wounds, wound healing and tissue engineering, Current problems in dermatology 31 (2003) 82-97.

[40] E. Wintermantel, J. Mayer, J. Blum, K.-L. Eckert, P. Lüscher, M. Mathey, Tissue engineering scaffolds using superstructures, Biomaterials 17(2) (1996) 83-91.

[41] J. Yip, S.-P. Ng, Study of three-dimensional spacer fabrics:: Physical and mechanical properties, Journal of Materials Processing Technology 206(1) (2008) 359-364.

[42] A.M. O'Mahony, J.L. Williams, J.O. Katz, P. Spencer, Anisotropic elastic properties of cancellous bone from a human edentulous mandible, Clinical oral implants research 11(5) (2000) 415-421.

[43] C.E. Misch, Z. Qu, M.W. Bidez, Mechanical properties of trabecular bone in the human mandible: implications for dental implant treatment planning and surgical placement, Journal of Oral and Maxillofacial Surgery 57(6) (1999) 700-706.

[44] R. Zhang, P.X. Ma, Poly (a-hydroxyl acids)/hydroxyapatite porous composites for bone-tissue engineering. I. Preparation and morphology, Journal of biomedical materials research 44(4) (1999) 446-455.

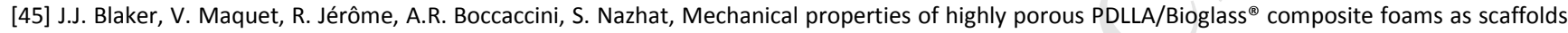
for bone tissue engineering, Acta biomaterialia 1(6) (2005) 643-652.

[46] J.F. Mano, Viscoelastic properties of bone: mechanical spectroscopy studies on a chicken model, Materials Science and Engineering: C 25(2) (2005) 145-152.

[47] Y. Liu, J. Lim, S.-H. Teoh, Review: development of clinically relevant scaffolds for vascularised bone tissue engineering, Biotechnology Advances 31(5) (2013) 688-705.

[48] V. Karageorgiou, D. Kaplan, Porosity of 3D biomaterial scaffolds and osteogenesis, Biomaterials 26(27) (2005) 5474-5491.

[49] D.W. Hutmacher, J.T. Schantz, C.X.F. Lam, K.C. Tan, T.C. Lim, State of the art and future directions of scaffold-based bone engineering from a biomaterials perspective, Journal of Tissue Engineering and Regenerative Medicine 1(4) (2007) 245-260.

[50] X. Wang, C. Han, X. Hu, H. Sun, C. You, C. Gao, Y. Haiyang, Applications of knitted mesh fabrication techniques to scaffolds for tissue engineering and regenerative medicine, Journal of the Mechanical Behavior of Biomedical Materials 4(7) (2011) 922-932.

[51] V.E. Santo, A.R.C. Duarte, E.G. Popa, M.E. Gomes, J.F. Mano, R.L. Reis, Enhancement of osteogenic differentiation of human adipose derived stem cells by the controlled release of platelet lysates from hybrid scaffolds produced by supercritical fluid foaming, Journal of Controlled Release 162(1) (2012) 19-27.

[52] S.V. Dorozhkin, Bioceramics of calcium orthophosphates, Biomaterials 31(7) (2010) 1465-1485.

[53] L.C. Palmer, C.J. Newcomb, S.R. Kaltz, E.D. Spoerke, S.I. Stupp, Biomimetic systems for hydroxyapatite mineralization inspired by bone and enamel, Chemical reviews 108(11) (2008) 4754-4783.

[54] E. Fiorentini, D. Granchi, E. Leonardi, N. Baldini, G. Ciapetti, Effects of osteogenic differentiation inducers on in vitro expanded adult mesenchymal stromal cells, International Journal of Artificial Organs 34(10) (2011) 998.

[55] B. Kundu, R. Rajkhowa, S.C. Kundu, X.G. Wang, Silk fibroin biomaterials for tissue regenerations, Advanced drug delivery reviews 65(4) (2013) 457470.

[56] Z. Ma, M. Kotaki, T. Yong, W. He, S. Ramakrishna, Surface engineering of electrospun polyethylene terephthalate (PET) nanofibers towards development of a new material for blood vessel engineering, Biomaterials 26(15) (2005) 2527-2536.

[57] H.K. Webb, J. Arnott, R.J. Crawford, E.P. Ivanova, Plastic degradation and its environmental implications with special reference to poly (ethylene terephthalate), Polymers 5(1) (2012) 1-18.

[58] I. Dal Pra, G. Freddi, J. Minic, A. Chiarini, U. Armato, De novo engineering of reticular connective tissue in vivo by silk fibroin nonwoven materials, Biomaterials 26(14) (2005) 1987-1999.

[59] J. Sanders, S. Bale, T. Neumann, Tissue response to microfibers of different polymers: polyester, polyethylene, polylactic acid, and polyurethane, Journal of biomedical materials research 62(2) (2002) 222-227.

[60] K. Illers, H. Breuer, Molecular motions in polyethylene terephthalate, Journal of Colloid Science 18(1) (1963) 1-31. 


\section{Supplementary data}
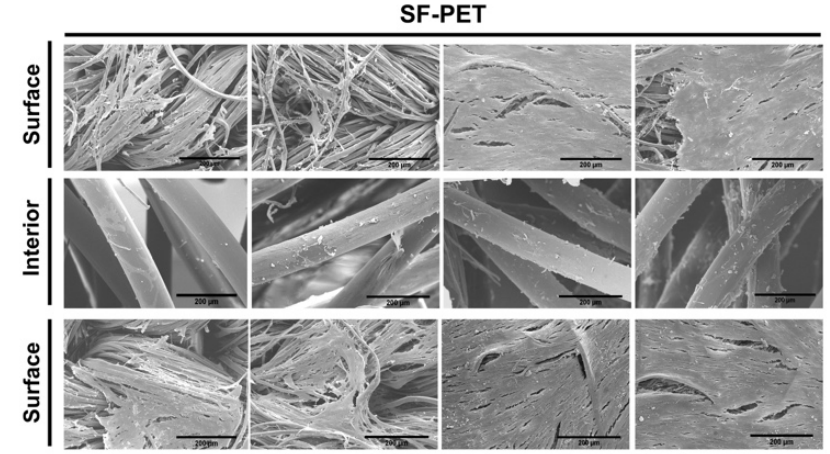

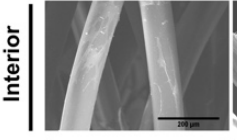

Day 7

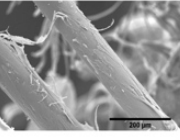

Day 14

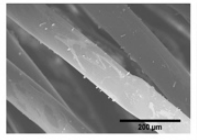

Day 21

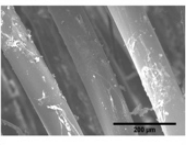

Day 28

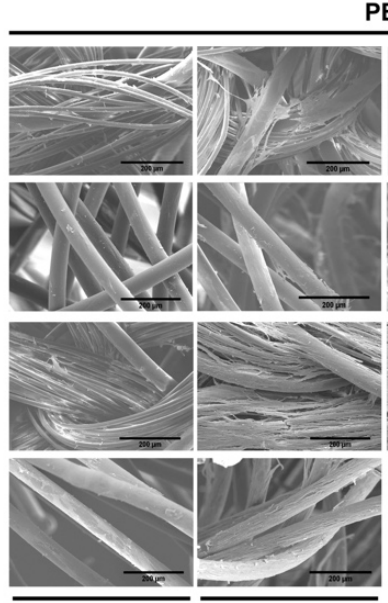

Day 7
PET

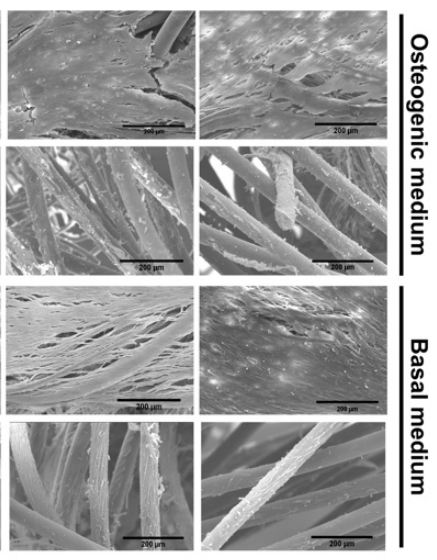

Day 21

Day 28

Supplementary Figure 1. SEM micrographs of the SF-PET and PET knitted spacer fabrics, cultured with hASCs in osteogenic and basal medium for 7, 14, 21 and 28 days. The figures show cell adhesion at the surface and cross-sections of the seeded scaffolds.

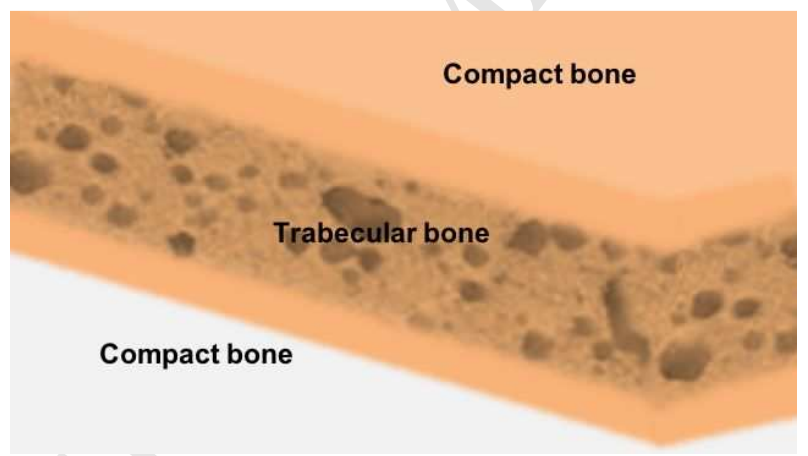

Supplementary Figure 2. Representative image of the structure of a flat bone. 